\title{
Probleme bei der Planung von Abwasseranlagen
}

\author{
Von A. Hörler, Dipl.-Ing. \\ Chef der technischen Abteilung der EAWAG, Zürich
}

\section{Einleitung}

Der Begriff «Planung» umfasst ein solch vielschichtiges Wissensgebiet, dass man im Rahmen eines Vortrages höchstens das Gesamtthema beleuchten kann. Selbst wenn diese Planung auf den Sektor Abwassertechnik beschränkt wird, ist das Gebiet, das die Planung umfasst, noch so vielfältig, dass unmöglich auf alle bei der Projektierung von Abwasseranlagen sich bietenden Probleme eingegangen werden kann. Es scheint mir auch, dass dem Ingenieur, der sich mit der Planung abzugeben hat, mit allgemeinen Gesichtspunkten nicht gedient wäre. Vielmehr dürfte es für ihn interessant sein, neben allgemeinen Erfahrungen auch Erörterungen über gewisse Detailfragen zu vernehmen, die immer wieder Anlass zu Meinungsverschiedenheiten geben. Es soll deshalb im nachfolgenden versucht werden, neben allgemeinen Gesichtspunkten namentlich einige spezielle Planungsprobleme zu erörtern und einer Lösung nahezubringen.

\section{Allgemeine Gesichtspunkte}

Der Ingenieur ist es gewohnt, zuerst durch Messungen und Erhebungen Unterlagen zu beschaffen, die ihm das Ausarbeiten eigentlicher Projekte durch Anwendung seiner statischen und hydraulischen Kenntnisse sowie der uibrigen Ingenieurwissenschaften erlaubt. Im Gegensatz zur Projektierung steht die Planung, bei der zusätzlich die möglichst genaue Erfassung der Entwicklung von Siedlungsgebieten und der Veränderung der Bedürfnisse der Wohnbevölkerung und der Industrien im Laufe von Jahrzehnten verlangt wird. Neben der Anwendung rationaler Wissenschaften, die objektive Beurteilungen erlauben, spielen die Intuition sowie gefühlsmässige und deshalb subjektive U̇berlegungen und Abschätzungen bei der Planung 
eine überragende Rolle. Dies mag auch der Grund dafür sein, dass sich der Planung im allgemeinen vorwiegend Architekten zugewendet haben, wodurch, meines Erachtens wenigstens, gefühlsbetonte Momente oft ein Übergewicht erhalten. Gerade bei der Planung muss seitens der Planenden nicht nur architektonisches Gefuihl vorhanden sein, sondern neben dem Einfühlungsvermögen in die Gegebenheiten auch weitgehende Kenntnisse der Bedürfnisse des Strassenbaus, des Verkehrs, der öffentlichen Betriebe, kurz des gesamten Siedlungswesens. Dabei nimmt das Gebiet der Siedlungswasserwirtschaft, speziell die Abwasseranlagen, nur einen zwar kleinen, aber trotzdem lebenswichtigen Teil ein. Da ein Einzelner die für wichtigere Planungsarbeiten notwendigen umfassenden Kenntnisse kaum besitzen kann, stellt sich somit die Forderung, dass bei grösseren Planungsarbeiten von Anfang an neben Spezialisten der anderen Sondergebiete auch ein Abwasserfachmann zugezogen werden sollte.

Für Planungen sind die unterschiedlichsten Gesichtspunkte massgebend. Prof. KeHR, Hannover, hat diese in einer Schrift: Standortfragen der Kläranlagen $[\mathrm{I}]^{1}$ ), nach ihrem Gewicht, wie nachfolgend angeführt, geordnet. Dabei scheint es mir, dass diese Anforderungen nicht unbedingt auf die Standortsfrage von Kläranlagen begrenzt zu sein brauchen, sondern dass sie eine universelle Bedeutung für die gesamten Abwasseranlagen besitzen.

Die Anforderungen von Prof. KeHR lauten wie folgt:

I. Hygienische Anforderung.

2. Wasserwirtschaftliche und entwässerungstechnische Anforderung (wobei ebenfalls die betriebstechnischen Gesichtspunkte Berücksichtigung finden sollen).

3. Städtebauliche A.nforderungen.

4. Wirtschaftliche Anforderungen.

5. Kommunalpolitische Erwägungen.

Aus dieser Zusammenstellung ist ersichtlich, dass die Wirtschaftlichkeit eines Planungsentwurfs nicht an erster Stelle steht oder stehen muss. Andere Gesichtspunkte können von wesentlicherer Bedeutung sein als die Wirtschaftlichkeit. Dies sowie der Umstand, dass Abwasseranlagen keine Rentabilität besitzen, wird der Grund dafür sein, dass häufig bei Planungen von Abwasseranlagen Wirtschaftlichkeitsberechnungen unterlassen oder nur in beschränktem Ausmasse durchgeführt werden.

Wie Barocka in seinem Buch über Wirtscbaftliche, organisatorische und

1) Die Ziffern in eckigen Klammern verweisen auf das Literaturverzeichnis, Seite 282. 
finanzielle Probleme des $W$ asserbaues und des Meliorationsmesens [2] eindrücklich erläutert hat, können Ortsentwässerungen ebenso wie Kläranlagen nicht allein und auch nicht in erster Linie nach Rentabilitätsgrundsätzen beurteilt werden. Ihr Bau kann aus hygienischen und allgemeinen wasserwirtschaftlichen Gründen dringend notwendig werden.

Mangelnde Rentabilität von Bauwerken darf aber niemals die Ursache dafür sein, Wirtschaftlichkeitsberechnungen zu unterlassen. Im Gegenteil! Gerade die mangelnde Rentabilität erfordert gründliche Wirtschaftlichkeitsvergleiche des gesamten Werkes oder einzelner Teile davon, zum mindesten jedoch eine Wirtschaftlichkeitsbeurteilung. Nur auf diese Weise kann oft die zweckmässigste Lösung gefunden werden. Eine Lösung kann dann als zweckmässig gelten, wenn mit sparsamsten Mitteln im ersten Ausbau ein maximaler Scbutz der ober- und unterirdischen Gewässer erzielt werden kann. Dies trifft häufig auch dann zu, wenn der Gesamtausbau des Werkes nicht die kleinste Kostensumme aufweist. Spätere Ausbauetappen dürfen dabei um so grössere Kosten bedingen, in je weiterer Ferne ihr Ausbau in Frage kommt. Es scheint, dass die Heranziehung des sogenannten Barwertes zur Beurteilung zukünftiger Bauausgaben ein zweckmässiges Mittel zur Erfassung der Wirtschaftlichkeit des Gesamtausbaues bietet.

Obgleich die Wirtschaftlichkeit in der Regel nicht an erster Stelle bei der Beurteilung eines Projektvorhabens steht, sollen im folgenden einige wirtschaftliche Erwägungen für bestimmte Fälle erläutert werden, da nur die Forderung der Wirtschaftlichkeit auf Grund von Vergleichsberechnungen gestattet, für bestimmte, allgemein gültige Annahmen ebenfalls bestimmte Aussagen zu machen. Sämtliche übrigen Anforderungen sind von den Lokalverhältnissen abhängig, die so verschiedenartig sind, dass sich nur allgemein gehaltene Richtlinien hierüber aufstellen lassen.

\section{Räumliche Abgrenzung des Planungsgebietes}

Als erste Aufgabe der Planung gilt es, das Planungsgebiet im Raum abzugrenzen. Diese Abgrenzung des Planungsgebietes sollte in erster Linie nach topographischen und nicht nach gemeindepolitischen Gesichtspunkten erfolgen. Massgebend sind somit die Wasserscheiden. Das eigentliche Kanalisationsgebiet, das mit dem gesamten Baugebiet zusammenfällt, ist in der Regel nur ein Ausschnitt aus dem Planungsgebiet, das als Einzugsgebiet bezeichnet werden kann. Dabei sollte es zur Selbstverständlichkeit werden, dass auch die Hanggebiete ausserhalb der eigentlichen Kanalisations- oder Baugebiete hydraulisch mitberücksichtigt werden, sofern nicht 
angenommen werden darf, dass längs den Baugebietsgrenzen Abfangkanäle für das Hang- und Sickerwasser angeordnet werden. Entsprechend grössere Anlauf- oder Retentionszeiten sind bei der Berechnung und Dimensionierung der Kanalisation zu berücksichtigen, wobei sich rechnerisch ergibt, dass die Hanggebiete nur einen wesentlichen Einfluss auf die Dimensionen der Endstränge haben. Dabei muss sich der Planer stets vor

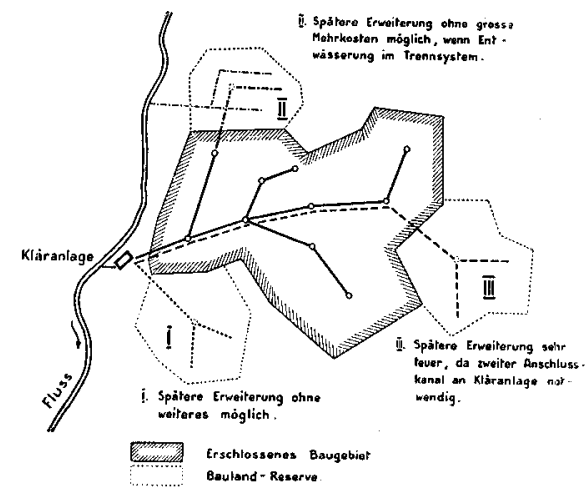

Abbildung 1

Entwässerungsgebiet mit drei Varianten für nachträgliche Baugebietserweiterung.

Augen halten, dass die Baugebiete selbst rechtskräftiger Bauzonenpläne je nach Bedarf abgeändert werden können. Kanalisationen können jedoch in den seltensten Fällen solchen Erweiterungen der Baugebiete ohne wesentliche finanzielle Belastungen der Gemeinden den neuen Verhältnissen angepasst werden. Es ist deshalb nicht nur zu begrüssen, sondern meines Erachtens ein dringendes Erfordernis, dass der Abwasserfachmann, wie bereits erwähnt, schon bei der Planung der Bauzonen herangezogen wird und dass die abwassertechnischen Gesichtspunkte bei der Ortsplanung mitberïcksichtigt werden. Zwei Bilder mögen dies erläutern.

Abbildung I entstammt einem Aufsatz von Ing. P. WILDI [3]. Daraus ist ersichtlich, wie zukünftige Erweiterungen von Baugebieten, vom kanalisationstechnischen Standpunkt aus betrachtet, zu bewerten sind.

Abbildung 2 zeigt ein Baugebiet, dessen Erschliessung verschieden erfolgen kann. Zudem wurde angenommen, dass das für die Industrie reservierte Baugebiet entweder oberhalb oder unterhalb des eigentlichen Wohngebietes angeordnet werden kann. Je nach der Wahl der Erschliessungsstrassen und der Anordnung der Industriegebiete können sich Verhältnisse 
ergeben, die bezüglich der Kanalisation wirtschaftlich weittragende Folgen nach sich ziehen können. Die mit «schlecht» bezeichnete Lösung wider-

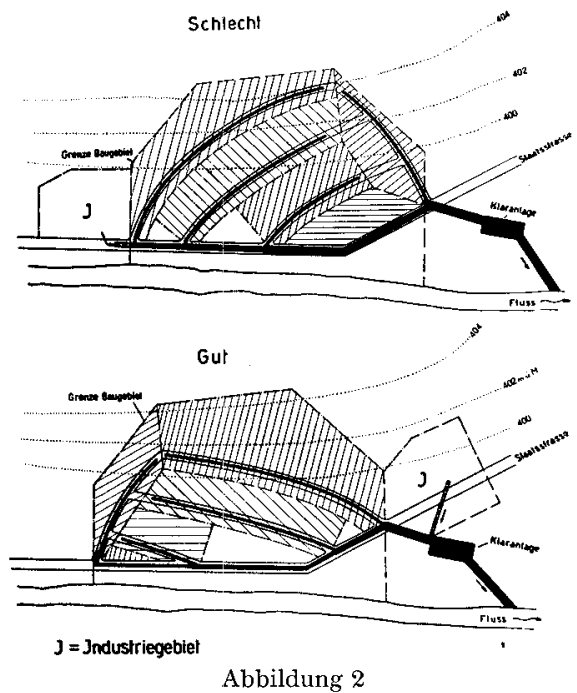

Kanalisationstechnisch schlechte (teure) und gute Lösung der Ortsentwässerung gleicher Siedlungsgebiete.

spricht dem Grundsatz der Kanalisationstechnik, dass das anfallende Abwasser so rasch als möglich aus dem Bereich der menschlichen Siedlung abgeleitet wird, da dies in vorliegendem Fall auf Umwegen geschieht, was kostenverteuernd wirkt.

\section{Vorstudien und Erhebungen}

Soll als Resultat der Planung ein Vorschlag resultieren, der die Zustimmung der zuständigen Behörden und des Bauherrn findet und als Grundlage aller weiteren Detailarbeiten dient, so muss den Vorstudien und Erhebungen, also den Ermittlungen der Grundlagen, auf denen sich das Gesamtprojekt aufbaut, grösste Beachtung geschenkt werden.

Solche Vorstudien und Erhebungen dürfen sich nicht auf die heute im Einzugsgebiet vorhandenen Einwohner beschränken. Der Projektverfasser soll sich auch Rechenschaft über die Bevölkerungsbewegung verschaffen, die für die nächsten Jahrzehnte bis zur Vollüberbauung des Einzugsgebietes als massgebend zu betrachten ist. Auf diese Weise können zweckmässige Vorschläge über den etappenweisen Ausbau von Abwasserreinigungs- 
anlagen, eventuell auch von Hauptsammelkanälen, sowie über den späteren Einbau von Regenwasserklärbecken bei Regenwasserentlastungen im Kanalisationsnetz gemacht werden.

Neben Ermittlungen der Bevölkerungsbewegung im Siedlungsgebiet und Ưberlegungen über die Entwicklung der gewerblichen und industriel-

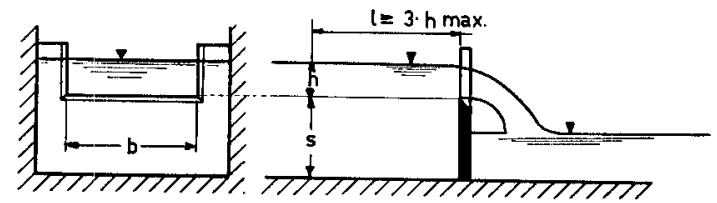

(1) NACH PONCELET

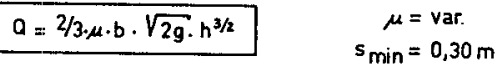

(NACH SIA. NORM 109)

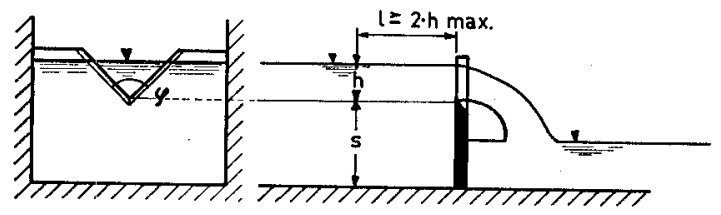

(2) NACH THOMSON (SPEZ. FÜR KLEINES Q)

$$
\begin{array}{ll}
0=\frac{8}{15} \cdot \mu \cdot \operatorname{tg} \frac{\varphi}{2} \cdot \sqrt{2 g} \cdot h^{5 / 2} \quad \text { FÜR } \varphi & =90^{\circ} \\
a & =1.40 \cdot \mathrm{h} 5 / 2 \\
5 & =0.40 \mathrm{~m}
\end{array}
$$

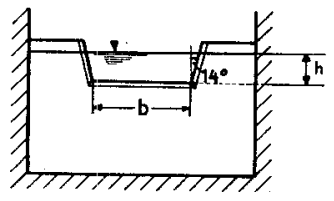

(3) NACH CIPPOLETTI

$$
\begin{aligned}
& Q=2 / 3 \cdot \mu \cdot b \cdot \sqrt{2 g} \cdot h^{3 / 2} \\
& \mu=0,632=\text { KONSTANT } \\
& Q=1,87 \cdot b \cdot h^{3 / 2}
\end{aligned}
$$

Abbildung 3

Überfallwehre für Wassermessungen.

len Betriebe des Planungsgebietes ist die Kenntnis des Abwasseranfalles und dessen Zusammensetzung von ausschlaggebender Bedeutung. Erste Erhebungen können durch Ermittlung des Wasserverbrauchs im betrachteten Einzugsgebiet gemacht werden. Immerhin ist zu beachten, dass der Wasserverbrauch mit dem Abwasseranfall nicht identisch sein kann. Ein- 
wandfreie Ergebnisse liefern Messungen des Abwasseranfalles in den Kanälen selbst. Zur Messung von Abwassermengen eignen sich nicht durchweg die nämlichen Messeinrichtungen wie für Reinwasser. Der bydrauliscbe Flügel zum Beispiel ist für Messzwecke ungeeignet wegen des Geschwemmsels, das vom Abwasser mitgeführt wird. Überfallmehre nach Abbildung 3 (Rechteckwehr nach Poncelet, dreieckiger Überfall nach THOMSON oder Trapezwehre nach CIPPOLETTI [4]) sind nur dann geeignet, wenn im Einzugsgebiet Hauskläranlagen vorhanden sind. Selbst dann noch wurde festgestellt, dass im Verlaufe von Wochen der durch den Messwehreinbau bedingte Stauraum verschlammte und damit die Messergebnisse beeinflusste. Wasserspiegelmessungen in Kanälen geben nur dann befriedigende Messresultate, wenn längs der Meßstrecke Normalabfluss vorhanden ist.

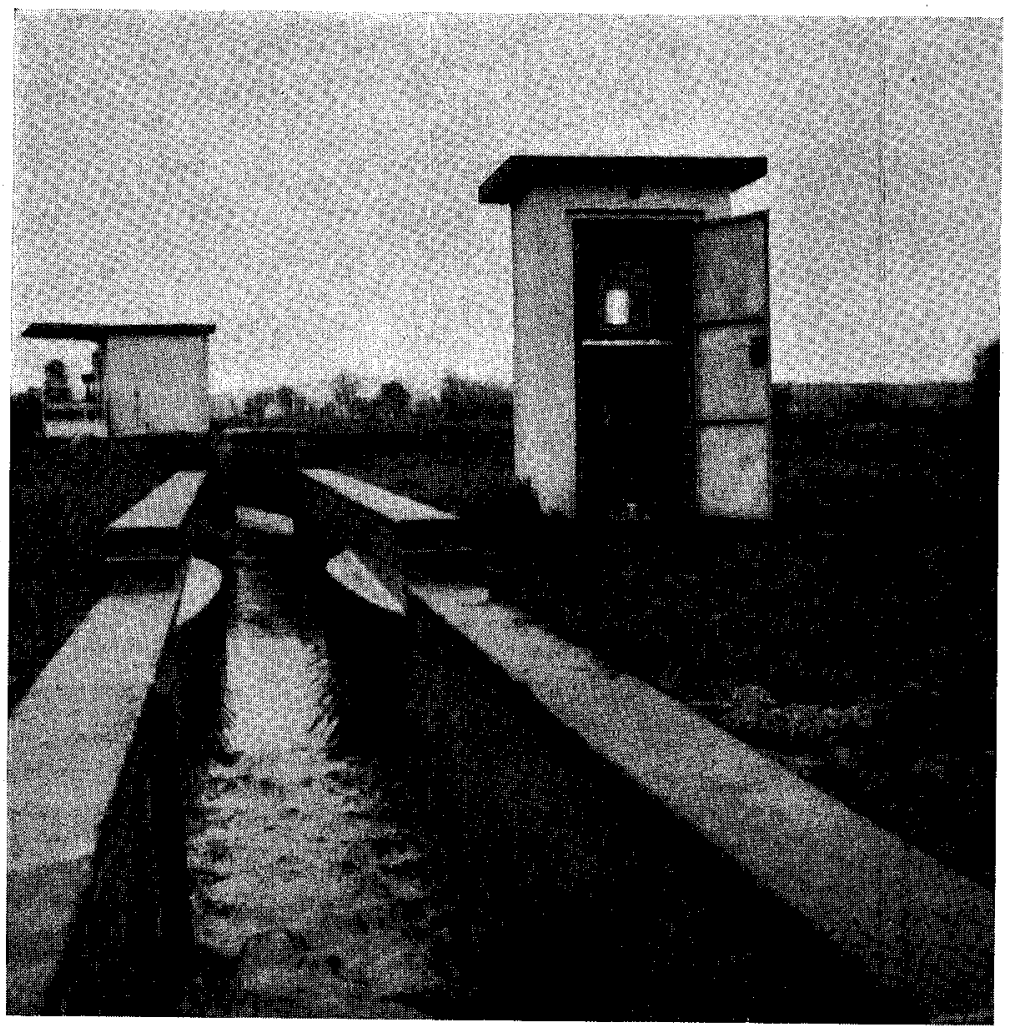

Abbildung 4

Kanal-Venturi-Messer der Kläranlage Langenthal (von unten gesehen). 


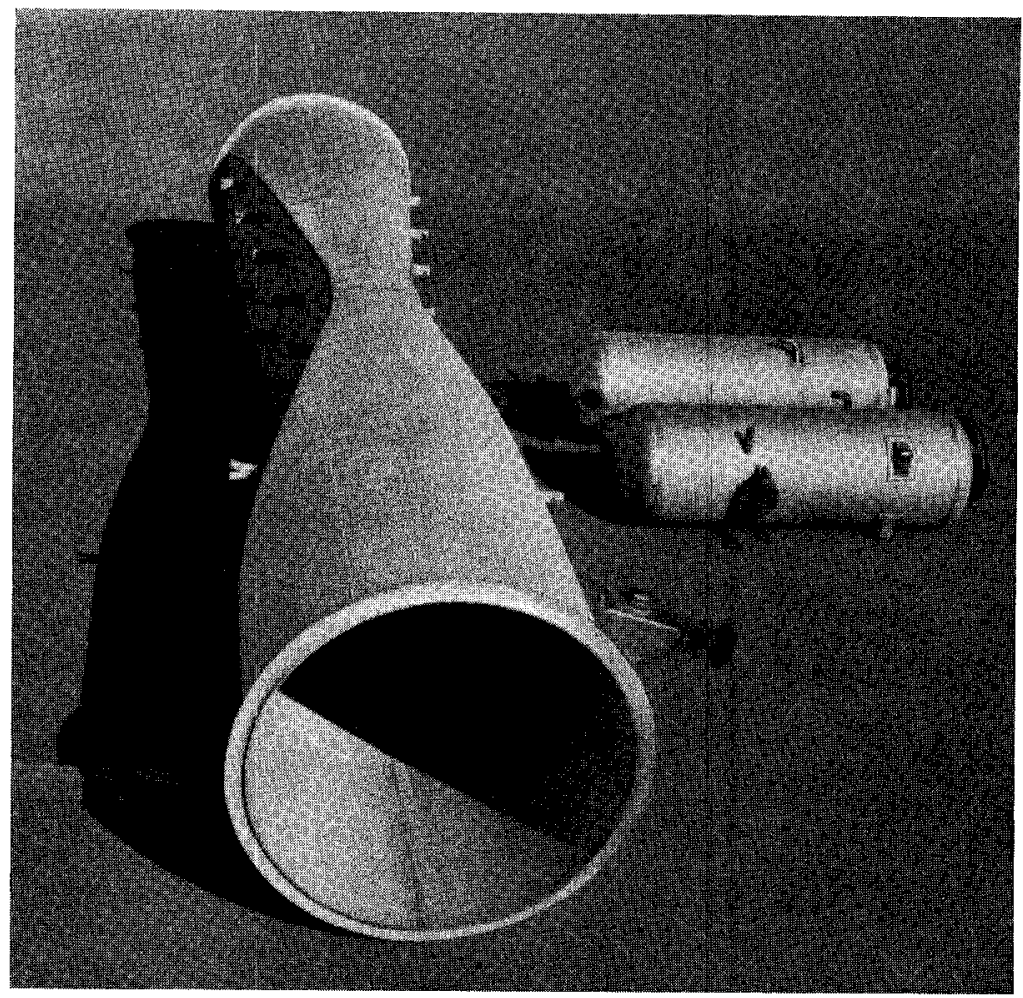

Abbildung 5

Venturi-Rohr der Druckleitung des Abwasserpumpwerkes Altstetten-Albisrieden der Stadt Zürich (Spezialkonstruktion für Differenzdruckmessung für Abwasser der Firma Rittmeyer AG, Zug).

Selbst dann ergibt jedoch die Schätzung des Rauhigkeitsbeiwertes sowie die Anwendung der Füllungskurve zur Ermittlung der Wassermenge eine Fehlerquelle. Zweckmässig haben sich hingegen zur Messung von Abwasser sogenannte Venturi-Kanäle (Abb. $\left.4^{2}\right)$ ) erwiesen, die durch die Erzwingung eines Übergangs vom Strömen zum Schiessen einwandfreie Wasserspiegellagen, unabhängig von den Unterwasserständen, ergeben. Für Wassermessungen in Druckröhren eignen sich speziell geformte Venturi-Robre, die sowohl den Sinkstoffen längs des Bodens als auch den Schwimmstoffen und Luftblasen im Rohrscheitel einen ungehinderten

2) Die Abbildungen 4-6 wurden mir in verdankenswerter Weise von der Firma Rittmeyer AG, Zug, die Abbildungen 7-9 von der Firma Züllig, Rheineck, zur Verfügung gestellt. 
Durchfluss ermöglichen. Die Abbildungen 5 und 6 zeigen ein solches Venturi-Rohr bzw. dessen Kern aus rostfreiem Stahl für die Druckleitung des Abwasserpumpwerkes in Zürich-Altstetten. In den USA sind weitere Messvorrichtungen gebräuchlich, die jedoch ebenfalls auf dem VenturiPrinzip beruhen, so der Parshall Flume [5], der Palmer-Bowlus Flume [6], die Staukörper [7] und die Kennizon Nozzle, welch letztere speziell zur Messung von flüssigem Klärschlamm geeignet sein soll.

Die Übersicht und Auswertung der Registrierstreifen von Wassermengenschreibern wird wesentlich erleichtert, wenn die Aufzeichnung der Wassermenge in einem linearen Maßstab erfolgt. Dies ist durch den Einbau sogenannter Kurvenscheiben in die Registrierapparate möglich (Abb. 7). Kombinierte Regen- und Abwassermengenschreiber, die ein wesentliches Hilfsmittel zur Erzielung einwandfreier Projektunterlagen sind, stehen ebenfalls zur Verfügung (Abb. 8 und 9).

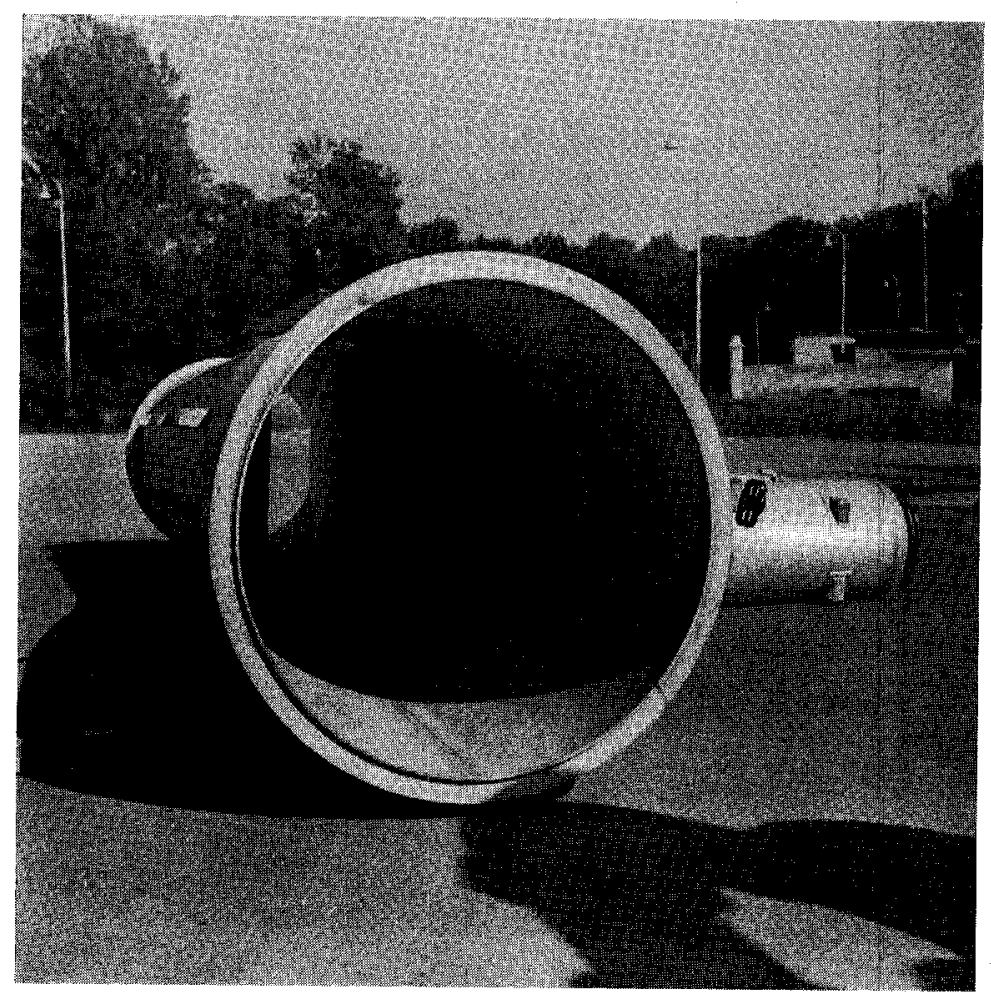

Abbildung 6

Wie Abbildung 5, jedoch Rohreinschnürung in Rohrachse gesehen. 


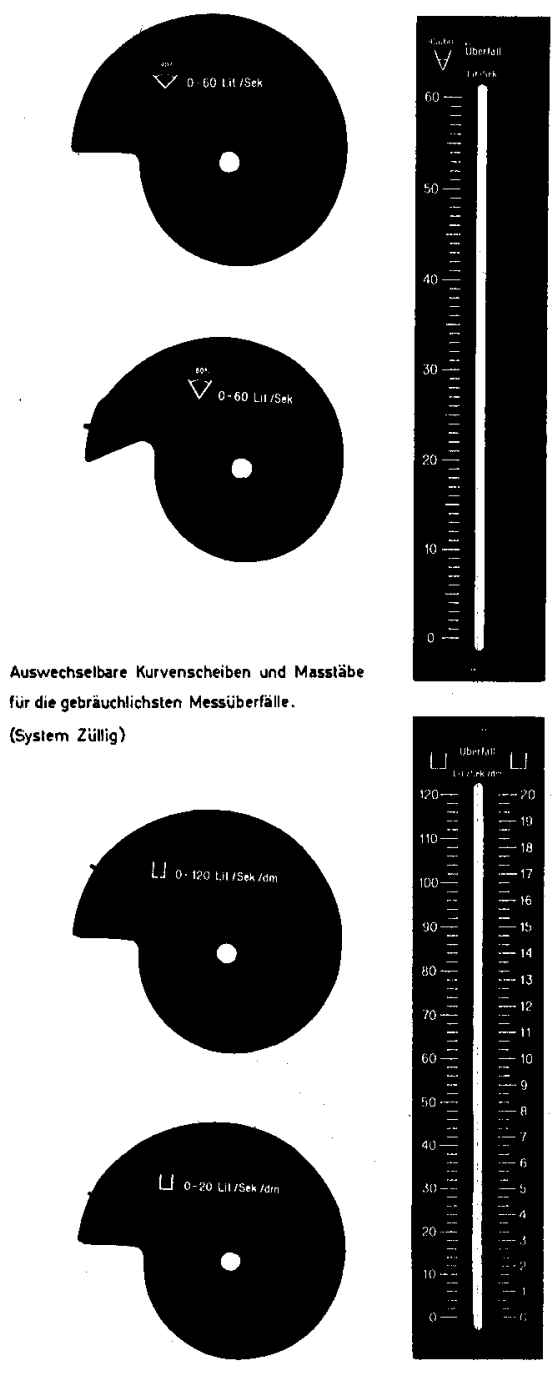

Abbildung 7

Auswechselbare Kurvenscheiben und Maßstäbe für Thomson- und Poncelet-Überfälle. 


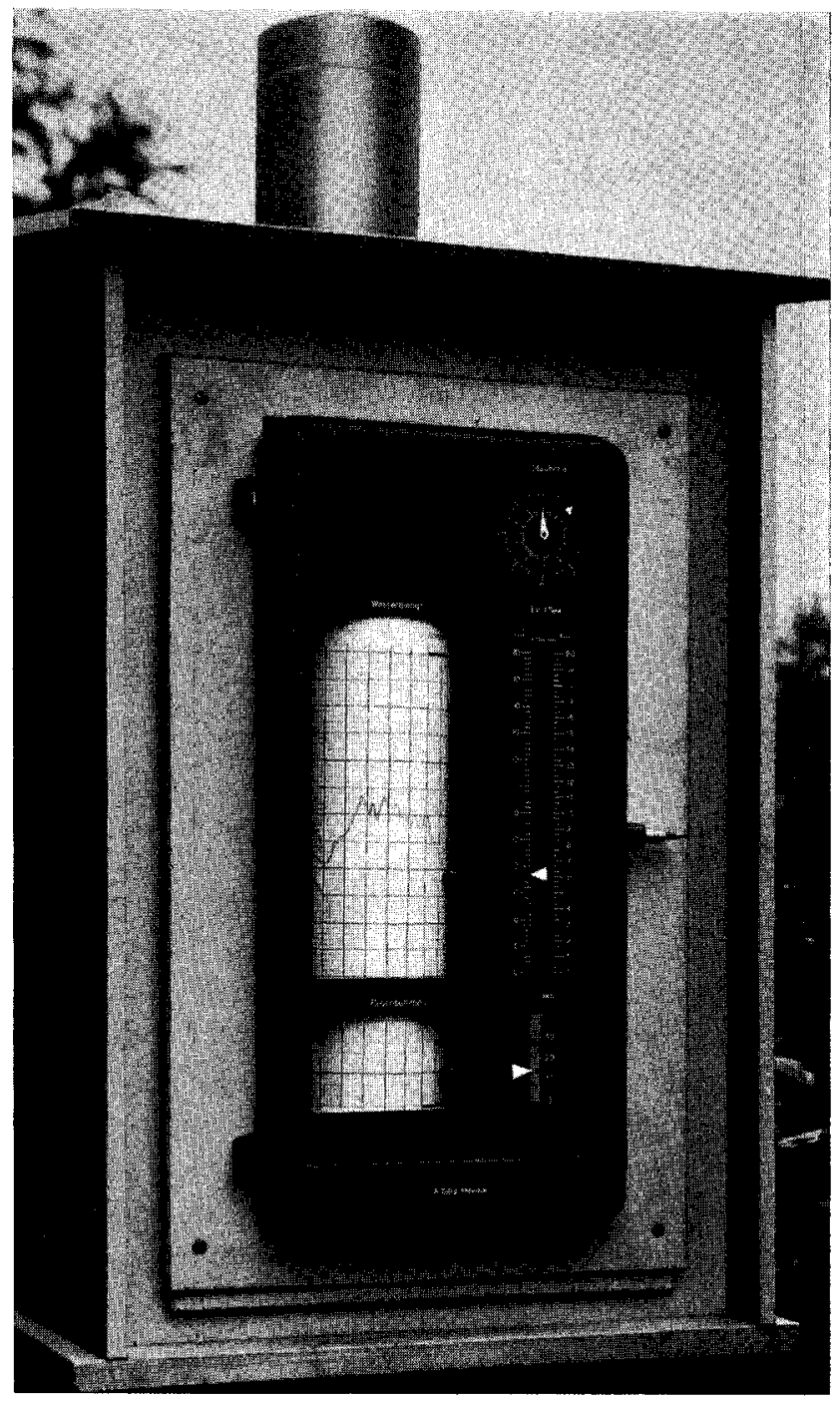

Abbildung 8

Kombinierter Regen- und Abwassermengenschreiber. 


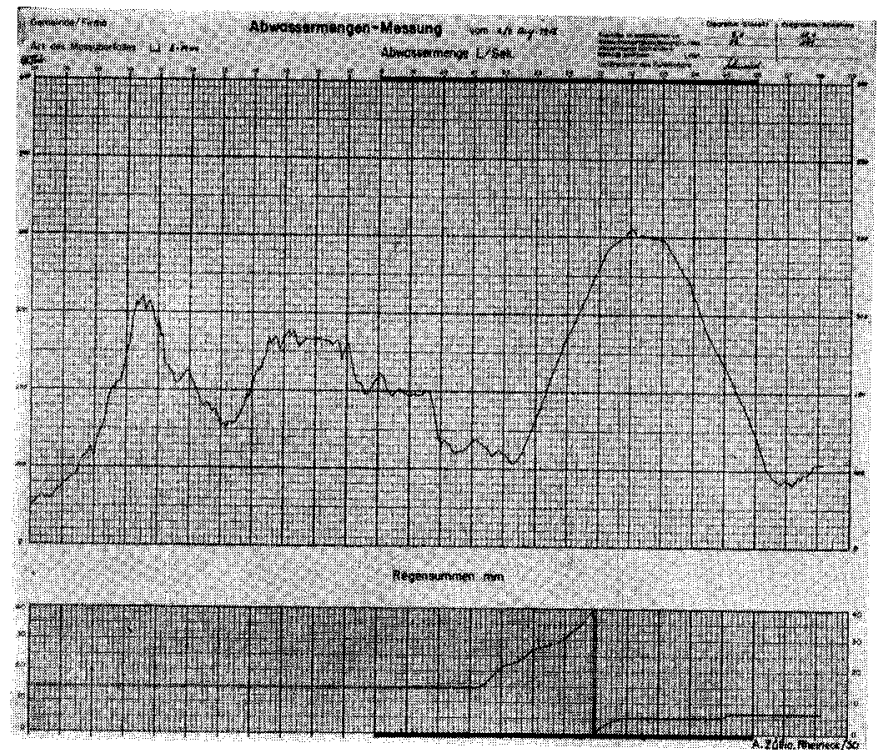

Abbildung 9

Regensummenkurve und Abflussdiagramm eines kombinierten Regen- und Abwassermengenschreibers.

Die Beziehung zwischen den Wasserständen, den Kanaleinschnürungen und den Wassermengen von Kanal-Venturi-Messern (Abb. Io) wurden eingehend von KAFAGHI [8] an der Versuchsanstalt für Wasserbau der Eidgenössischen Technischen Hochschule untersucht. Leider sind die Versuchsresultate in einer für den praktisch tätigen Ingenieur nicht gerade ansprechenden Form veröffentlicht, die langwierige, wenn auch keinesfalls komplizierte Zwischenrechnungen erfordern. Es zeigt sich jedoch, dass sich die Gleichung für die Wassermenge auf folgende einfache Form bringen lässt, deren Genauigkeit für Abwassermengenmessungen genügt:

$$
Q=1,71 t^{3 / 2} b_{2} h_{1}{ }^{3 / 2},
$$

wobei $Q$ Wassermenge in Kubikmetern pro Sekunde; $t=H: h_{1}=$ Energielinienhöhe: Wassertiefe vor der Einschnürung; $b_{2}$ Kanalbreite in der Einschnürung in Metern; $h_{1}$ Wassertiefe oberhalb der Meßstelle in Metern.

Für bestimmte Einschnürungsverhältnisse lassen sich die Werte $I, 7 \mathrm{I} t^{3 / 2}$ zu einem Beiwert $\mu$ vereinigen, so dass dann die vereinfachte Formel wie folgt lautet:

$$
Q=\mu b_{2} h^{3 / 2},
$$



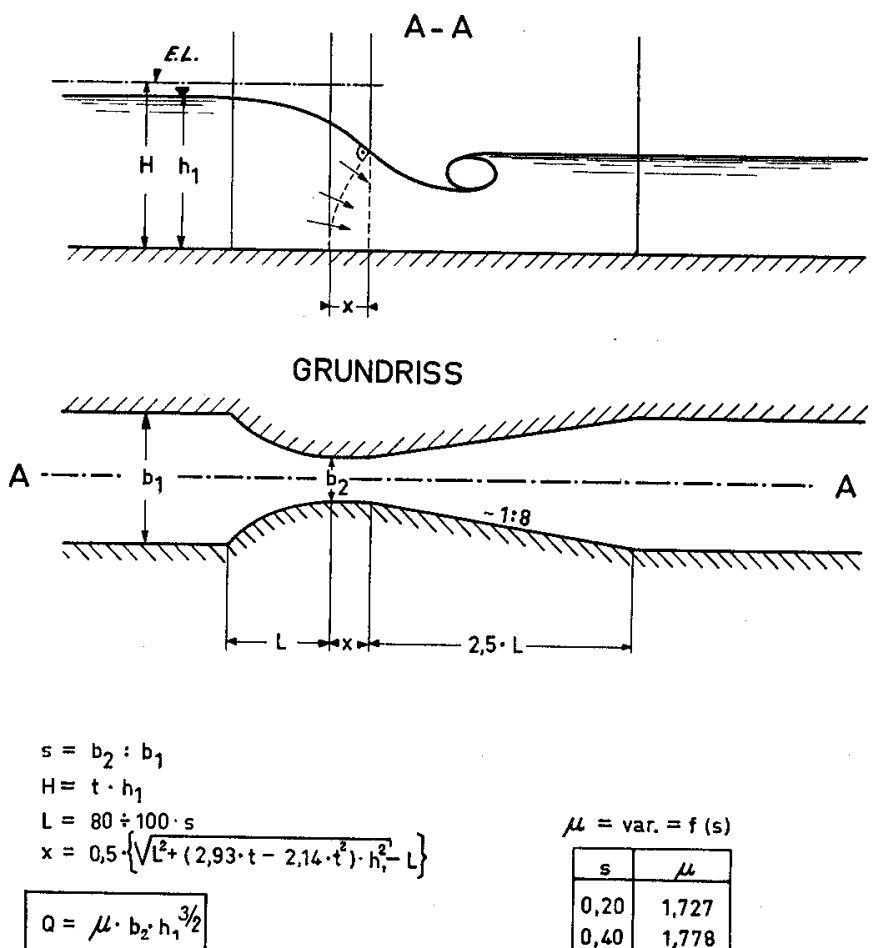

$\mu=$ var. $=f(s)$
\begin{tabular}{|c|c|}
\hline$s$ & $\mu$ \\
\hline 0,20 & 1,727 \\
0,40 & 1,778 \\
0,60 & 1,880 \\
0,80 & 2,076 \\
\hline
\end{tabular}

Abbildung 10

Venturi-Kanal für Wassermengenmessung nach KAFAGHr.

wobei für verschiedene Einschnürungsverhältnisse $s=b_{2}: b_{1}=$ Breite in der Einschnürung: Breite oberhalb der Meßstelle, der Wert $\mu$ aus folgender Tabelle entnommen werden kann:

\begin{tabular}{|c|c|}
\hline$s$ & $\mu$ \\
\hline 0,25 & 1,736 \\
0,30 & 1,747 \\
0,35 & 1,761 \\
0,40 & 1,778 \\
0,45 & 1,798 \\
0,50 & 1,820 \\
0,55 & 1,847 \\
0,60 & 1,880 \\
0,65 & 1,919 \\
0,70 & 1,964 \\
0,75 & 2,016 \\
\hline
\end{tabular}


Unter Verwendung der Gleichung (2) und obenstehender Tabelle ist die Wassermenge leicht zu ermitteln.

Über dieses gesamte Problem der Messung von Abwassermengen hat Oberingenieur VON DER EMDE, Hannover, einen bemerkenswerten Aufsatz [9] veröffentlicht, auf den hiermit verwiesen sei.

Der stark veränderlichen Abwasserzusammensetzung wegen ist eine eingehende Erhebung des industriellen Abwasseranfalles unerlässlich. Zur ersten Orientierung sind die von H. F. KurseL, Chemiker der EAWAG, herausgebrachten Fragebogen äusserst wertvoll.

So wertvoll die Erhebungen über den Anfall und die Zusammensetzung des Abwassers eines Siedlungsgebietes sind, so darf doch nicht ausser acht gelassen werden, dass damit die für die Planung wesentliche Arbeit noch nicht geleistet ist. Leider werden hie und da die Messergebnisse unbedenklich den weiteren Planungsarbeiten zugrunde gelegt. Es dürfte jedoch selbstverständlich sein, dass diese Messergebnisse nur Mittel zum Zweck sein können, einem Zweck, der darin besteht, den zukünftigen Abwasseranfall und dessen Zusammensetzung im voraus zu ermessen und dessen Entwicklung bis zur Vollüberbauung des Einzugsgebietes festzulegen. Diese letzterwähnten Ergebnisse sind ausschlaggebend für die Grösse der Abwasseranlagen und deren etappenweisen Ausbau.

\section{Wahl des Standortes der Kläranlagen, Einzel- oder Sammelreinigungsanlage}

\section{A. WAHL DES STANDORTES DER KLÄrANLAGE}

Über die Wahl des Standortes von Kläranlagen sind, in der Fachliteratur verstreut, vereinzelte Hinweise vorhanden ([2], [3], [Io], [rI]). Umfassendste Angaben in dieser Hinsicht veröffentlichte Prof. KeHR, Hannover, in seiner schon erwähnten Abhandlung über Standortfragen für Kläranlagen [I], auf die hiermit ausdrücklich hingewiesen sei. Prof. KEHR kommt dabei zu folgenden Ergebnissen, die hiermit im Auszug wiedergegeben seien.

\section{Hygienische Anforderungen}

Gut gebaute Kläranlagen sind dicht und haben deshalb keine Beeinträchtigung unterirdischer Gewässer zur Folge. Gut betriebene Kläranlagen weisen keine Geruchsbelästigungen auf. In der Nähe von Wohnsied- 
lungen ist das Belebtschlammverfahren dem Tropfkörperverfahren überlegen, da keine Psychodafliegen auftreten ${ }^{3}$ ).

Neben den hygienischen Gründen spielen auch ästhetische Belange eine wesentliche Rolle im Entscheid bezüglich der Standortswahl.

\section{Wasserwirtschaftliche und entwässerungstechnische Anforderungen}

Kläranlagen gehören an grosse Vorfluter, sofern diese mit tragbaren Mitteln erreicht werden können. Wesentlich ist die strömungstechnisch richtige Wahl der Abwassereinleitungsstelle in den Vorfluter. Namentlich bei biologischen Reinigungsanlagen ist eine Zusammenfassung der $\mathrm{Ab}$ wasser mehrerer Gemeinden in einer einzigen Reinigungsanlage erstrebenswert. Dies ist nicht nur technisch - namentlich betriebstechnisch besser, sondern häufig auch wirtschaftlicher.

\section{Städtebauliche Anforderung'en}

Da gut betriebene Kläranlagen geruchlos arbeiten, können diese im Baugebiet erstellt werden. Anderseits dürfen städtebaulich wichtige oder traditionsgebundene Grundstücke nicht mit Kläranlagen belegt werden, und zwar schon aus ästhetischen Gründen. Hingegen können dort in der Regel Abwasserpumpwerke errichtet werden, die das Abwasser auf städtebaulich weniger interessante Grundstücke fördern, wo eine Reinigungsanlage ohne Bedenken erstellt werden kann.

\section{Wirtscbaftliche Anforderungen}

Die Projektierung von Abwasserreinigungsanlagen soll nicht nur ingenieurtechnisch richtig erfolgen, sondern hat auch die wirtschaftlichen Erfordernisse zu berücksichtigen. Kostenvergleiche für die verschiedenen technisch möglichen Lösungen sind auszuarbeiten, bevor zur Standortsfrage Stellung genommen wird. Hierzu sei auf die nachfolgend unter B. aufgeführten Äusserungen verwiesen.

3) In Russland besteht die Vorschrift von Schutzzonen bei Wohnvierteln oder Anlagen der Lebensmittelindustrie. So hat zum Beispiel die Schutzzone zwischen Kläranlagen und Siedlungsgebiet bei Rieselfeldern $1 \mathrm{~km}$, bei allen andern Reinigungsarten mindestens $300 \mathrm{~m} \mathrm{zu}$ betragen. Die letzterwähnte Forderung dürfte bei den heutigen Abwasserreinigungsverfahren als überholt $\mathrm{zu}$ betrachten sein. 


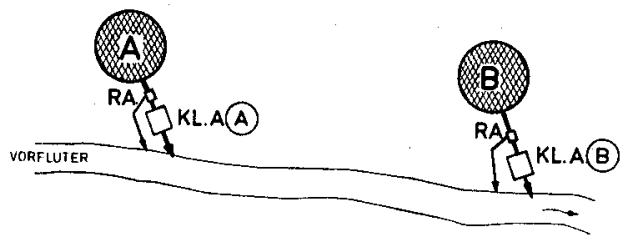

SAMMEL-ANLAGE

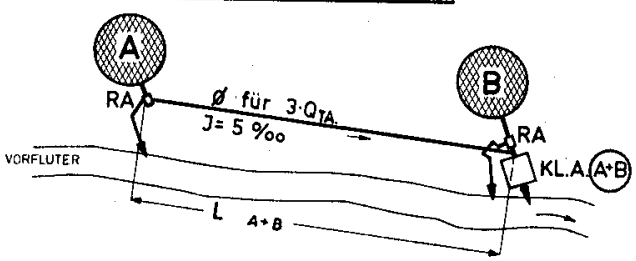

Abbildung 11

Schematische Darstellung von getrennten (Einzel-) bzw. Sammelreinigungsanlagen

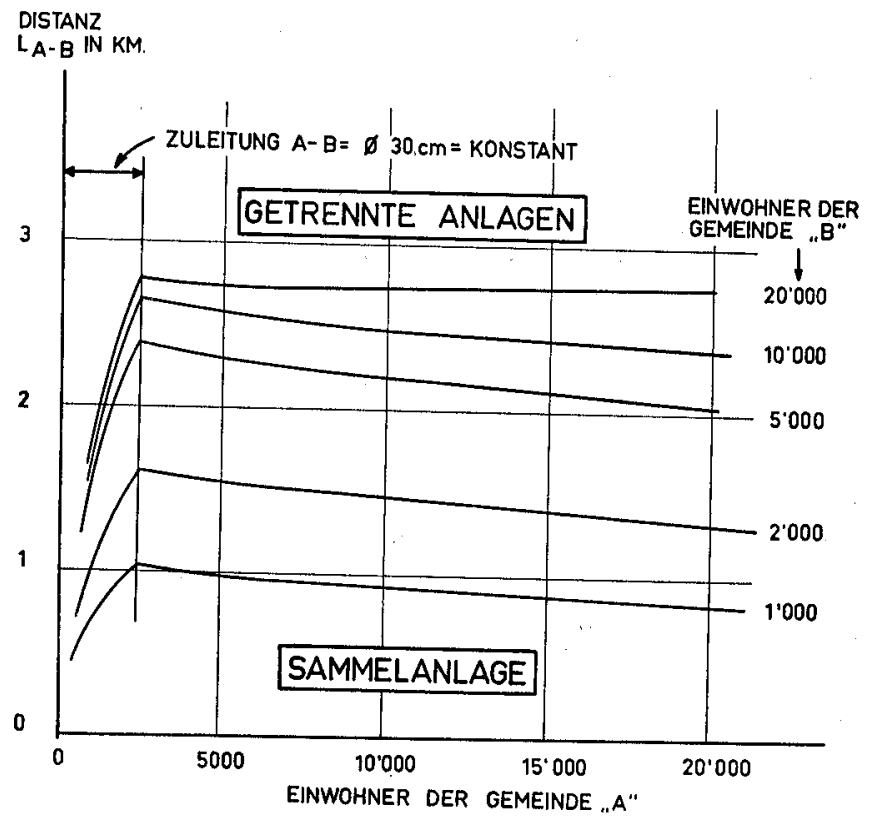

Abbildung 12

Wirtschaftlich gleichwertige Distanzen der Gemeinden $A$ und $B$ für getrennte bzw. Sammelkläranlagen, falls eine mechanische Klärung der Abwässer genügt. 


\section{Kommunalpolitische Erwägungen}

Siedlungswirtschaftliches Denken und Planen hat über die zufälligen politischen Gemeindegrenzen hinauszugehen. Die Furcht vor einer «Eingemeindung» veranlasst häufig kleinere Gemeinden, einer Gemeinschaftslösung Widerstand entgegenzusetzen. Gemeinschaftslösungen können jedoch ohne weiteres durchgeführt werden, ohne dass eine Eingemeindung erfolgen muss.

\section{B. EINZEL- ODER SAMMELREINIGUNGSANLAGEN}

Allgemeingültige Schlüsse lassen sich namentlich auf Grund wirtschaftlicher Erwägungen machen. So dürfte es von besonderem Interesse sein, diejenigen Abstände von Gemeinden kennenzulernen, die, vom rein wirtschaftlichen Standpunkt aus betrachtet, als Grenzabstand gewertet werden können, bei dem ein Zusammenschluss noch verantwortet werden kann. Als Beispiel wurden zwei Gemeinden einer Talschaft gewählt, wobei die oberhalb liegende mit $A$, die unterhalb liegende Gemeinde mit $B$ bezeichnet wurde (Abb.II). Das Gefälle des Verbindungskanals beider Gemeinden wurde zu $\zeta^{\%} \%$ angenommen. Für beide Gemeinden wurden als alternative Ausbauziele der Klär- bzw. Abwasserreinigungsanlagen 1000, 2000, 5000, I0000 und 20000 angeschlossene Einwohner gewählt. Nimmt man vorerst Einzelanlagen für jede Gemeinde an und ermittelt deren Baukosten sowie deren kapitalisierte Betriebskosten, so wird man feststellen, dass die Summe beider Einzelanlagen der Gemeinden $A$ und $B$ auf jeden Fall eine grössere Kapitalaufwendung erfordert als eine Sammelreinigungsanlage für beide Gemeinden zusammen. Dividiert man den Differenzbetrag beider Lösungen durch den Preis pro laufenden Meter des bei der Sammelreinigungsanlage erforderlichen Verbindungskanals der Gemeinden, so erhält man denjenigen Abstand beider Gemeinden, bei welchem die beiden Lösungen - Einzelanlagen bzw. Sammelreinigungsanlagen - wirtschaftlich gleichwertig sind. Diese wirtschaftlich gleichwertigen Distanzen sind in den Abbildungen I 2 und I 3 graphisch dargestellt. Dabei ist aus Abbildung $\mathbf{2} 2$ die wirtschaftlich gleichwertige Distanz ersichtlich, falls mechanische Kläranlagen zur Reinigung des Abwassers genügen, während der Abbildung I 3 die Annahme zugrunde liegt, dass das Abwasser biologisch gereinigt werden muss.

Aus diesen Abbildungen geht hervor, dass ein Zusammenschluss um so interessanter ist, je grösser die Gemeinde $B$ gegenüber der Gemeinde $A$ 
ist, und dass er um so mehr angestrebt werden muss, je grösser die verlangte Reinigungsleistung der Abwasserreinigungsanlage ist.

Zudem ist aus den Abbildungen generell ersichtlich, dass bei grösseren Gemeinden $B$ ein Zusammenschluss wirtschaftlich dann noch interessant ist, wenn die Distanz 2-3 km beträgt im Falle mechanischer Klärung bzw. $3-5 \mathrm{~km}$, falls biologische Teilreinigung gefordert wird.

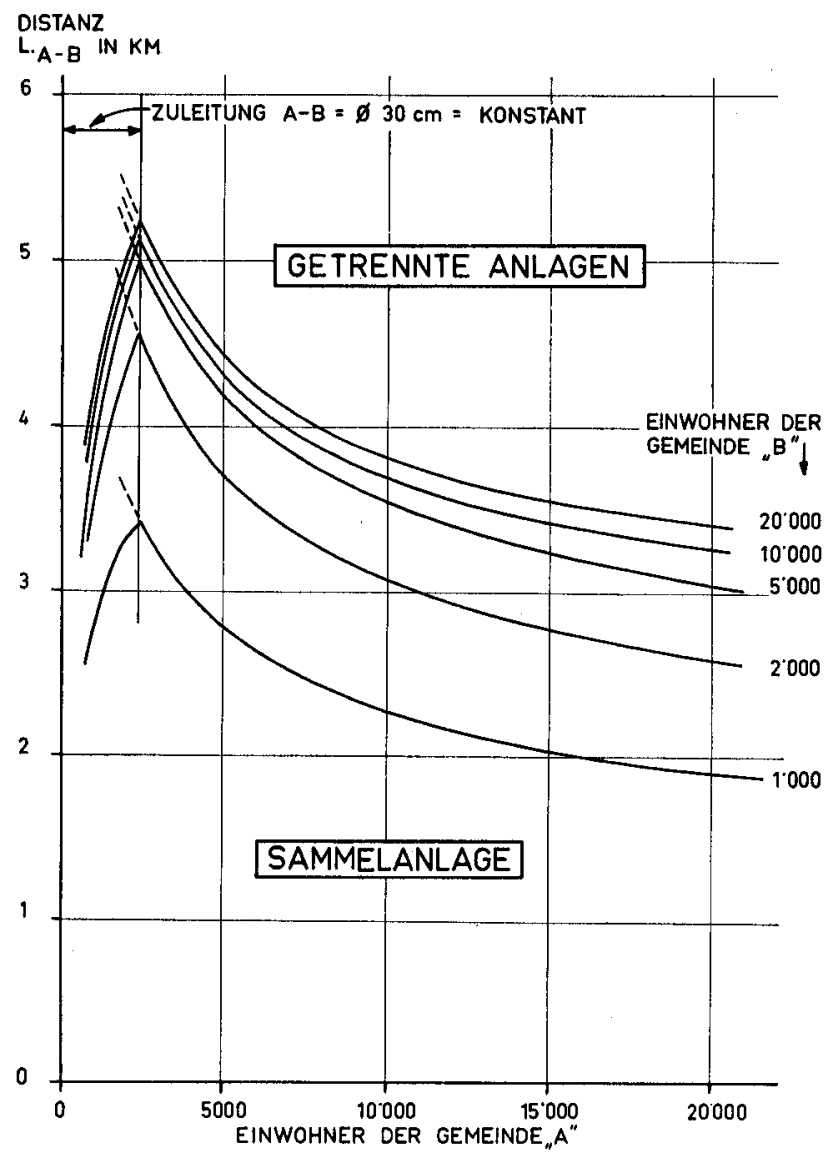

Abbildung 13

Wirtschaftlich gleichwertige Distanzen $\operatorname{der}$ Gemeinden $A$ und $B$ für getrennte bzw. Sammelreinigungsanlagen, falls eine biologische Teilreinigung erforderlich ist. 


\section{Trennsystem oder Mischsystem}

A. VOR- UND NACHTEILE DER BEIDEN SYSTEME

Die Vor- und Nachteile der beiden Systeme wurden mehr oder weniger vollkommen in der Literatur wiederholt veröffentlicht ([Io], [I2], [I3], [I4]). Nachfolgend sei eine ergänzte Aufstellung dieser Vor- und Nachteile, die jedoch keinen Anspruch auf Vollständigkeit erhebt, wiedergegeben, ohne dass im Detail auf einzelne Faktoren näher eingetreten wird.

\begin{tabular}{|c|c|c|}
\hline Gesichtspunkte, Objekt & Trennsystem & Mischsystem \\
\hline \multicolumn{3}{|c|}{ a) Wirtschaftliche Gesichtspunkte } \\
\hline & 2 Kanäle in jeder Strasse. & Nur 1 Kanal erforderlich. \\
\hline $\begin{array}{l}\text { Unterhalt, Spülung, } \\
\text { Reinigung }\end{array}$ & Teurer. & Billiger. \\
\hline $\begin{array}{l}\text { Kosten des } \\
\text { Sammelkanals }\end{array}$ & $\begin{array}{l}\text { In Tiefgebieten günsti- } \\
\text { ger, sofern Regenwasser } \\
\text { in hochliegenden Kanälen } \\
\text { direkt an den Vorfluter } \\
\text { übergeben werden kann. } \\
\text { Dem Regenwasserkanal } \\
\text { kann ein grösseres Ge- } \\
\text { fälle gegeben werden. }\end{array}$ & $\begin{array}{l}\text { Häufig wesentlich gerin- } \\
\text { gere Kosten. }\end{array}$ \\
\hline $\begin{array}{l}\text { Viele kleine Wasserläufe } \\
\text { im Einzugsgebiet }\end{array}$ & $\begin{array}{l}\text { Vorteilhaft, da Regen- } \\
\text { wasser in hochliegenden, } \\
\text { kurzen Kanälen diesen } \\
\text { Bachläufen übergeben } \\
\text { werden kann. }\end{array}$ & $\begin{array}{l}\text { Zur Anordnung der Re- } \\
\text { genauslässe liegen die } \\
\text { Hochwasserspiegel die- } \\
\text { ser Bachläufe oft zu } \\
\text { hoch, so dass die gesam- } \\
\text { ten Regenwassermengen } \\
\text { grössere Strecken abge- } \\
\text { leitet werden müssen. }\end{array}$ \\
\hline Kläranlagen & $\begin{array}{l}\text { Kühlwasser von Indu- } \\
\text { strien können an. die } \\
\text { Regenwasserleitungen an- } \\
\text { geschlossen werden, eben- } \\
\text { so Abläufe der Laufbrun- } \\
\text { nen. Dadurch wird die } \\
\text { Kläranlage entlastet. }\end{array}$ & $\begin{array}{l}\text { Diese dürfen bei Regen- } \\
\text { wetter etwas überlastet } \\
\text { werden, so dass deren } \\
\text { Ausbildung nicht wesent- } \\
\text { lich teurer wird als beim } \\
\text { Trennverfahren, sofern } \\
\text { Reinwasseranteil gering } \\
\text { ist. }\end{array}$ \\
\hline
\end{tabular}




\begin{tabular}{|c|c|c|}
\hline Gesichtspunkte, Objekt & Trennsystem & Mischsystem. \\
\hline \multicolumn{3}{|c|}{ a) Wirtschaftliche Gesichtspunkte } \\
\hline Hausanschlüsse & $\begin{array}{l}\text { Teurer; die doppelte } \\
\text { Hauskanalisation für Re- } \\
\text { genwasser und Schmutz- } \\
\text { wasser getrennt erfor- } \\
\text { derlich. }\end{array}$ & Einfacher und billiger. \\
\hline \multicolumn{3}{|c|}{ b) Technische Gesichtspunkte } \\
\hline $\begin{array}{l}\text { Rückstau bei } \\
\text { Platzregen }\end{array}$ & $\begin{array}{l}\text { Rückstau bei Platzregen } \\
\text { hat keine Kellerüber- } \\
\text { schwemmungen zur Fol- } \\
\text { ge. Ausnahme: Rückstau } \\
\text { via Hausdrainagen. }\end{array}$ & $\begin{array}{l}\text { Kellerüberschwem- } \\
\text { mungen möglich. }\end{array}$ \\
\hline $\begin{array}{l}\text { Rückstau bei Hochwasser } \\
\text { des Vorfluters }\end{array}$ & do. & $\begin{array}{l}\text { Je nach gegenseitiger } \\
\text { Höhenlage von Keller- } \\
\text { boden und Hochwasser- } \\
\text { stand im Vorfluter mög- } \\
\text { lich. }\end{array}$ \\
\hline Trockenwetterabfluss & $\begin{array}{l}\text { Gute Spülwirkung im } \\
\text { Schmutzwassernetz, da } \\
\text { relativ grosse Wasser- } \\
\text { tiefe. }\end{array}$ & $\begin{array}{l}\text { Kleine Abwassertiefe und } \\
\text { geringere Schleppkraft. }\end{array}$ \\
\hline $\begin{array}{l}\text { Bei agressivem } \\
\text { Abwasser }\end{array}$ & $\begin{array}{l}\text { Als Material für die } \\
\text { Schmutzwasserleitung } \\
\text { können Steinzeugrohre } \\
\text { verwendet werden. }\end{array}$ & $\begin{array}{l}\text { Kanalquerschnitt ist mei- } \\
\text { stens so gross, dass eine } \\
\text { Verwendung von Stein- } \\
\text { zeug als Kanalprofil } \\
\text { nicht in Frage kommt. } \\
\text { Oft werden Sohleinlagen } \\
\text { aus Steinzeug eingelegt, } \\
\text { die gegen Aggressivität } \\
\text { jedoch nur einen be- } \\
\text { schränkten Schutz ge- } \\
\text { währen, sofern nicht ein- } \\
\text { wandfrei verfugt. }\end{array}$ \\
\hline $\begin{array}{l}\text { Schmutzwasser- } \\
\text { Pumpstationen }\end{array}$ & $\begin{array}{l}\text { Gleichmässige Beanspru- } \\
\text { chung der Pumpen. }\end{array}$ & $\begin{array}{l}\text { Neben den Schmutzwas- } \\
\text { serpumpen sind grosse } \\
\text { Einheiten zur Förderung } \\
\text { des Regenwassers not- } \\
\text { wendig, die nur relativ } \\
\text { wenige Stunden pro Jahr } \\
\text { in Tätigkeit sind. Kost- } \\
\text { spielige Stationen. }\end{array}$ \\
\hline
\end{tabular}




\begin{tabular}{|c|c|c|}
\hline Gesichtspunkte, Objekt & Trennsystem & Mischsystem \\
\hline \multicolumn{3}{|c|}{ b) Technische Gesichtspunkte } \\
\hline $\begin{array}{l}\text { Beanspruchung des } \\
\text { Strassenkörpers }\end{array}$ & $\begin{array}{l}\text { In schmalen Strassen oft } \\
\text { schwierig, } 2 \text { Kanäle neben } \\
\text { den übrigen Werkleitun- } \\
\text { gen unterzubringen. }\end{array}$ & Günstiger. \\
\hline \multicolumn{3}{|c|}{ c) Hygienische Gesichtspunkte } \\
\hline Regenauslässe & $\begin{array}{l}\text { kommen nicht vor (dafür } \\
\text { Strassenabschwemmung, } \\
\text { evtl. von Mistwürfen). }\end{array}$ & $\begin{array}{l}\text { Belasten den Vorfluter } \\
\text { erheblich, so dass oft } \\
\text { Regenwasserkläranlagen } \\
\text { vorgesehen werden müs- } \\
\text { sen (trotzdem nur Be- } \\
\text { helfsmassnahme). }\end{array}$ \\
\hline $\begin{array}{l}\text { Bei schwachen Regen- } \\
\text { fällen mit Intensitäten } \\
\text { bis etwa } 151 / \mathrm{s} \text { ha (rund } \\
90 \% \text { der jährlichen } \\
\text { Regendauer) }\end{array}$ & $\begin{array}{l}\text { Strassenschmutz wird } \\
\text { durch Regenwasserkanäle } \\
\text { in den Vorfluter gespült. } \\
\text { Um dies zu verhindern, } \\
\text { kann eine Verbindung } \\
\text { zwischen Regen- und } \\
\text { Schmutzwasserleitung } \\
\text { angeordnet werden, dass } \\
\text { erste, kleine Regenmen- } \\
\text { gen in die Schmutzwas- } \\
\text { serleitung gelangen. }\end{array}$ & $\begin{array}{l}\text { Diese Regen bewirken } \\
\text { noch kein Spiel der Re- } \\
\text { genauslässe zur Kläran- } \\
\text { lage und belasten somit } \\
\text { den Vorfluter nicht (Aus- } \\
\text { nahme: evtl. Notauslass } \\
\text { vor Kläranlage). }\end{array}$ \\
\hline
\end{tabular}

\section{B. SYSTEMWAHL IN DEN EINZELNEN LÄNDERN}

Wir stellen die Frage: «Welches System ist bei der Projektierung von Abwasseranlagen zu wählen, das Trenn- oder das Mischsystem?» In England und in den USA, vorwiegend jedoch in Russland und Polen, wird fast durchweg nach dem Trennsystem kanalisiert, während in Deutschland und namentlich in der Schweiz das Mischsystem im Vordergrund steht. Wieso dieser Unterschied? Betrachten wir die Verhältnisse in England, den USA und Russland, die zur Wahl des Trennsystems Anlass geben, so ist festzustellen, dass es sich dabei fast durchweg um Flachgebiete handelt mit mangelnder Entlastungsmöglichkeit. Durch die Wahl des Trennsystems ist es oft möglich, die hochliegenden Regenwasserleitungen direkt in die Vorfluter einzuführen, ohne dabei gezwungen zu sein, das gesamte Abwasser, also das Schmutz- und Regenwasser, gemeinsam zu pumpen. 
Dieser Umstand allein schon mag die Wahl des Trennsystems begründen. Hingegen ist in hügeligem Gelände mit Entlastungsmöglichkeit das Mischsystem gegenüber dem Trennsystem wirtschaftlich dermassen überlegen, dass in der Regel keine Zweifel über die Wahl des Systems auftreten können. Ob das Trennsystem grössere hygienische Vorteile gegenüber dem Mischsystem aufweist, ist, entgegen der gefühlsmässigen Beurteilung, zum mindesten zweifelhaft. Selbst Regenwasser aus Wohnsiedlungen stellt eine Belastung dar, wie im nächsten Abschnitt dargelegt wird. Dies ist in vermehrtem Masse der Fall, wenn im Siedlungsgebiet landwirtschaftliche Anwesen mit Mistwürfen vorhanden sind. Wohl wird beim Mischsystem das Entlastungswasser eine höhere Konzentration aufweisen als das Regenwasser des Trennsystems. Durch die Wahl geeigneter Entlastungsverhältnisse kann jedoch erreicht werden, dass bei den einzelnen Regenwasserentlastungen nur etwa 10-20\% derjenigen Wassermasse in den Vorfluter gelangt, die bei einer Entwässerung im Trennsystem in den Vorfluter gelangen würde, so dass die Gesamtbelastung des Vorfluters bei Entwässerung nach dem Mischsystem kaum grösser ausfallen dürfte als bei Anwendung des Trennsystems.

\section{WaHl des ENTwässerungssystems für PUMPgebiete}

Selbst Fachleute vertreten oft die Meinung, dass Pumpgebiete im Trennsystem entwässert werden sollten, und zwar aus wirtschaftlichen Gründen. Da sich diese Auffassung sogar in der Fachliteratur vorfindet, sei mir gestattet, speziell auf diese Frage näher einzutreten.

Bei der Beurteilung der Frage, ob in Pumpgebieten Trenn- oder Mischsystem gewählt werden soll, sind drei getrennte Fälle zu unterscheiden:

$$
\text { Fall } A
$$

Die Kanalisation im Pumpgebiet liegt so tief unter dem Vorfluterwasserspiegel, dass selbst bei Anwendung des Trennsystems Schmutz- und Regenwasser gepumpt werden müssen.

\section{Fall $B$}

Die Kanalisation des Pumpgebietes liegt tiefer als der Vorfluterwasserspiegel, jedoch so, dass bei einer Entwässerung im Trennsystem eine Ableitung des Regenwassers mit freiem Gefälle gerade noch möglich ist, während die Anordnung von Regenauslässen bei Entwässerung im Mischsystem nicht verantwortet werden könnte. 


\section{Fall C}

Die Kanalisation des Pumpgebietes liegt genügend hoch über dem Vorfluterwasserspiegel, so dass Regenauslässe bei einer Entwässerung im Mischsystem vorgesehen werden können.

Fall $A$. In diesem Falle dürfte stets eine Entwässerung im Mischsystem in Frage kommen, da Schmutz- und Regenwasser gepumpt werden müssen.

Fall B. Sofern die Entwässerung des Gebietes im Mischsystem erfolgt, muss das gesamte Abwasser (Schmutzwasser und Regenwasser) gepumpt werden. Wird diese gesamte Abwassermenge einem Sammelkanal mit freiem Gefälle bis zur Kläranlage zugepumpt, dann erhält dieser Sammelkanal eine erhebliche zusätzliche Belastung bis zum nächstgelegenen Regenauslass.

In gewissen Fällen wären zwei Möglichkeiten für die Aufteilung der Pumpenaggregate in zwei funktionell verschiedenartige Aufgaben möglich: Eine Schmutzwasserpumpengruppe zur Förderung des 3-5fach « verdünnten» Schmutzwassers in einen höhergelegenen Sammelkanal und eine automatische Inbetriebsetzung einer Regenwasserpumpe bei stärkeren Regenfällen mit Förderung direkt in den höhergelegenen Vorfluter.

Da die Regenwassermenge oft das roofache des Schmutzwasseranfalles überschreitet, zeigt ein Wirtschaftlichkeitsvergleich, dass allein die Erstellungskosten des Mischwasserpumpwerkes eine Entwässerung im Trennsystem gegenüber dem Mischsystem zum mindesten nahelegen, abgesehen von den Betriebskosten, die beim Pumpen der gesamten Regenwassermasse noch hinzukämen, die jedoch nicht ausschlaggebend sein können. Erschwerend kommt hinzu, dass beim Pumpen des gesamten Regenwasseranfalles gewisse Pumpengruppen nur wenig in Betrieb sind und dass zudem genügend Reservegruppen und eine von der elektrischen Energiezuführung unabhängige Energieversorgung vorhanden sein sollte. Für den Fall $B$ ist somit eine Entwässerung des Pumpgebietes im Trennsystem in der Regel anzustreben.

Fall C. Für diesen Fall soll vorerst abgeklärt werden, ob es bei der Entwässerung im Mischsystem vorteilhafter ist, neben dem Trockenwetterabfluss noch einen Regenwasseranteil entsprechend der kritischen Intensität von $20 \mathrm{l} / \mathrm{sha}$ mitzufördern, oder ob zweckmässiger die Fördermenge auf den dreifachen Trockenwetteranfall begrenzt, dafür aber zusätzlich ein Regenwasserklärbecken erstellt wird. Für die Grösse dieses Beckens wurde 
eine Aufenthaltszeit von to Minuten bei einer Regenintensität von $20 \mathrm{l} / \mathrm{sh}$ a angenommen.

Zur Abklärung vorstehender Frage werden im folgenden für eine Wohnzone (65 E/ha, Abflussbeiwert 0,35 ) für verschiedene Flächengrössen der Pumpgebiete von I bis Ioo ha für die beiden Möglichkeiten die Kosten einander gegenübergestellt (Abb. I4).

Ein Vergleich der für die beiden Varianten aufgeführten generellen Kosten zeigt, dass die Variante mit Regenwasserklärbecken bei Gebieten

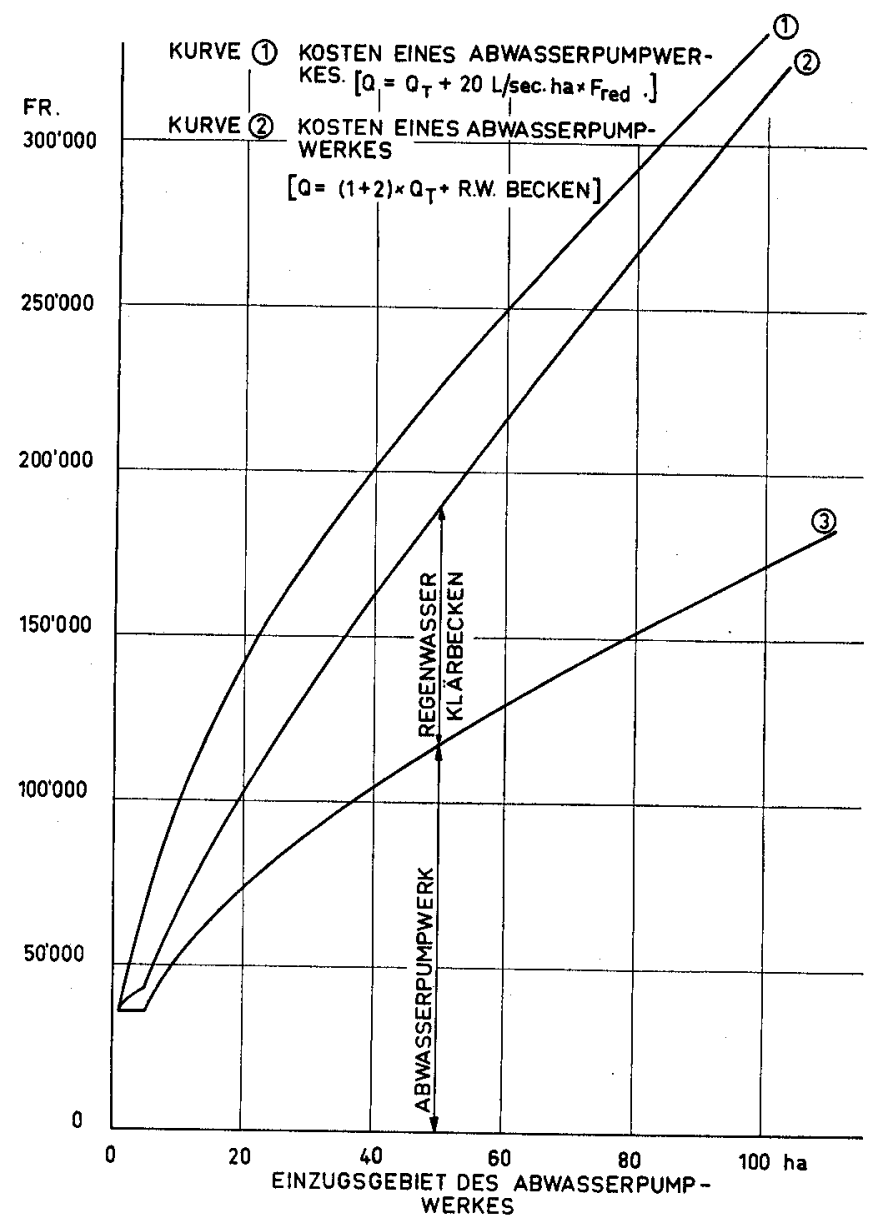

Abbildung 14

Kostenvergleich von Abwasserpumpwerken ohne und mit Regenwasserklärbecken. 
über I ha bezüglich der Baukosten günstiger ausfällt als die Variante ohne Regenklärbecken. Da die Druckleitungen der Variante ohne Regenklärbecken einen grösseren Durchmesser aufweisen, verschieben sich die Baukosten bei Berücksichtigung der Druckleitung weiterhin zugunsten der Variante mit Klärbecken. Zudem weist die letztere Variante kleinere Betriebskosten auf, da der zu pumpende Regenwasseranteil kleiner ist.

Als Resultat obiger Betrachtungen stellen wir fest, dass es in vorstehendem Beispiel wirtschaftlicher ist, die Fördermenge des Pumpwerkes auf

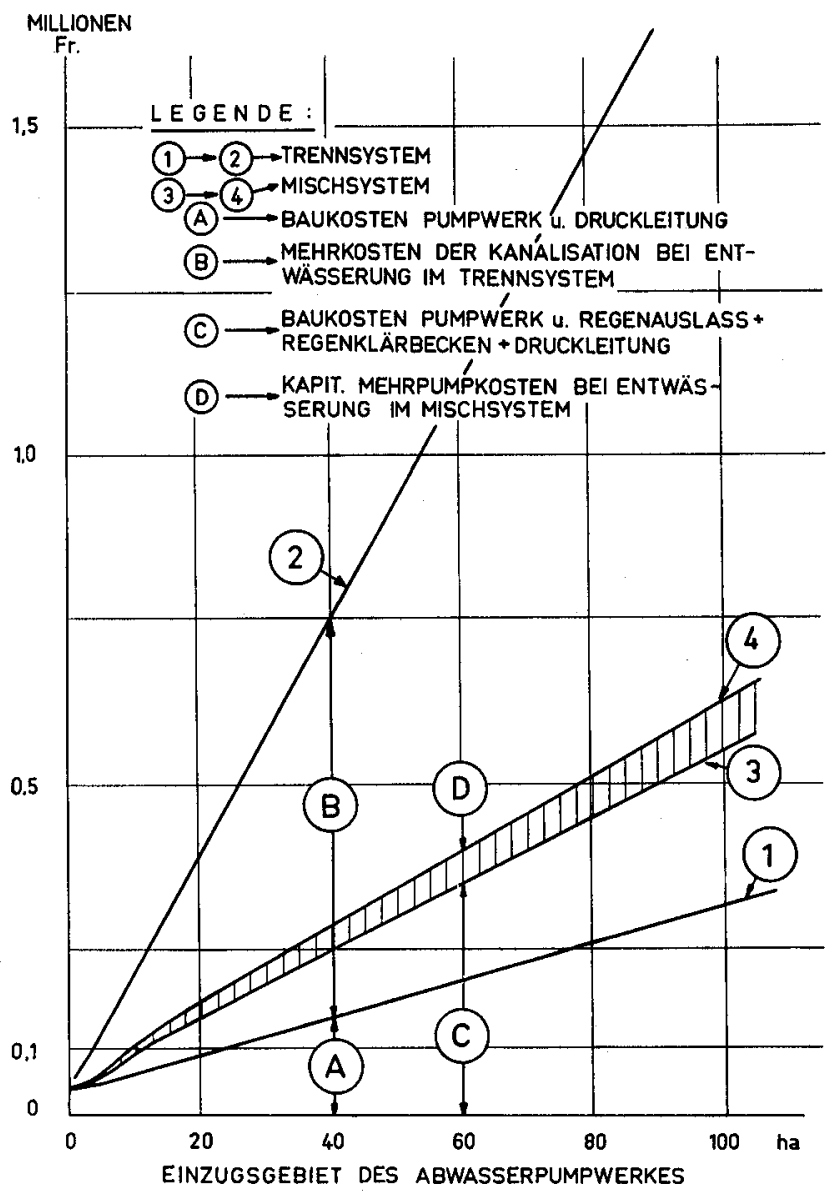

Abbildung 15

Kostenvergleich zwischen Entwässerung im Trennsystem (ohne Ableitung des Oberflächenwassers in offenen Gräben und mit beschränkter Einleitungsmöglichkeit von Regenwasser in Vorfluter) und Entwässerung im Mischsystem bei Pumpgebieten. 
den dreifachen Trockenwetteranfall zu begrenzen und ein Regenwasserklärbecken vorzusehen, als den Regenwasseranfall bis zur kritischen Regenintensität mitzufördern und dafür das Regenwasserklärbecken einzusparen.

Für die Abklärung der Hauptfrage, ob Mischsystem oder Trennsystem vorzusehen sei (in dem mit Fall $C$ bezeichneten Pumpgebiet), sind somit beim Mischsystem die Aufwendungen für die Variante mit Erstellung eines Regenwasserklärbeckens den Kosten des Trennsystems gegenüberzustellen.

Die Mehrkosten der Entwässerung eines Gebietes im Trennsystem mit der doppelten Kanalführung gegenüber der Entwässerung im Mischsystem sind von Fall zu Fall verschieden. Sie betragen im Beispiel pro Hektare Einzugsgebiet rund Fr. I5000.-. Die unter dieser Annahme errechneten approximativen Kosten für Gebietsgrössen von I bis Ioo ha der betrachteten Wohnzone sind in Abbildung Is dargestellt.

Aus dieser Abbildung geht hervor, dass die Baukosten bei Entwässerung im Mischsystem geringer sind als bei der Entwässerung im Trennsystem. Bei kleinen Gebieten ist der Unterschied nicht sehr ins Gewicht fallend. Bei grösseren Gebieten ist dieser Unterschied jedoch wesentlich. Bei Wahl des Mischsystems ist fallweise die Möglichkeit der Erstellung von Regenauslässen zu überprüfen. Als Entlastungsbauwerke kommen im vorliegenden Fall entweder Streichwehre in Frage, wobei die Überfallkante mindestens $10 \mathrm{~cm}$ hoch sein muss, oder eine Kombination der Entlastung mit dem Regenwasserklärbecken bzw. dem Pumpensumpf und möglichst hochgelegener Überfallkante. Die letztere Lösung ist, wenn technisch möglich, in der Regel die bessere.

Da die Betriebskosten für die Förderung des Trockenwetterabflusses samt Sickerwasser bei den beiden Varianten (Mischsystem und Trennsystem) praktisch gleich sind, wurden nur die Mehrkosten für das Pumpen des Regenwasseranteils (2mal Trockenwetteranfall) beim Mischsystem ermittelt und in der Abbildung I5 als kapitalisierte Betriebskosten schraffiert dargestellt.

Der Vergleich zeigt, dass selbst unter Berücksichtigung des beim Mischsystem zusätzlich zu pumpenden Regenwasseranteils eine Entwässerung nach dem Mischsystem, namentlich für Gebiete von I ha Fläche und mehr, wirtschaftlich bedeutend vorteilhafter ist.

Zusammenfassend kann somit gesagt werden, dass für die Gebiete $C$ (Pumpzonen, deren Kanäle über den höchsten Wasserspiegel des Vorfluters zu liegen kommen) in der Regel das Mischsystem vorzuziehen ist, sofern 
nicht vorerst nur die Schmutzwasserkanalisation erstellt wird oder das Regenwasser ganz oder teilweise in offenen Gräben abgeleitet werden kann. Auch bei Pumpgebieten, die sich längs einem zur Entlastung geeigneten Vorfluter hinziehen, kann eine Entwässerung im Trennsystem in Frage kommen. Wirtschaftlichkeitsvergleiche der verschiedenen Entwässerungsmöglichkeiten sind in solchen Fällen unerlässlich.

\section{Regenauslässe}

\section{A. ZWECK}

Regenauslässe sind Entlastungsbauwerke, die bei einer Entwässerung im Mischsystem an zweckmässig ausgewählten Punkten des Kanalisationsnetzes erstellt werden. Da die Regenwassermengen das Hundert- und Mehrfache der Schmutzwassermenge betragen können, wäre es wirtschaftlich nicht zu verantworten, bei grösseren Entwässerungsgebieten mit Entlastungsmöglichkeit in einen Vorfluter auch bei Starkregen die Gesamtregenmasse bis zur Kläranlage weiterzuleiten, die doch nicht für die Aufnahme und Reinigung der gesamten Abwassermenge dimensioniert werden könnte, ebenfalls aus wirtschaftlichen Gründen.

\section{B. ANWENDUNGSBEREICH}

Regenauslässe werden in der Regel dort angeordnet, wo der Hauptsammelkanal in die Nähe eines genügend leistungsfähigen Vorfluters gelangt. Verläuft der Hauptsammelkanal in unmittelbarer Nähe parallel zum Vorfluter, so werden in der Regel im Verlauf der Kanalisation mehrere Regenauslässe hintereinander angeordnet. Allgemein ist zu sagen, dass in solchen Fällen eher zu viel Regenauslassbauwerke erstellt werden, was unerwünscht ist. Aber auch aus wirtschaftlichen Gründen lässt sich die Anordnung vieler Regenauslässe häufig nicht begründen. Jeder Regenauslass benötigt als Sonderbauwerk zusätzliche Baukosten, die durch den erforderlichen Regenauslasskanal noch vermehrt werden. Werden diese zusätzlichen Ausgaben für die Erstellung grösserer Hauptsammelkanäle mit weniger, aber grösseren Regenauslässen an speziell geeigneten Stellen verwendet, so wird man oft bemerken, dass dadurch eine zweckmässigere Lösung ohne wesentlich grössere Gesamtkosten entsteht. Die Ursache dieses Resultates liegt darin begründet, dass ein Sekundenliter Abwasser bei gleichem Gefälle um so billiger transportiert wird, je grösser die Rohrleitun- 
gen sind. Wie aus Abbildung I6 ersichtlich, sind namentlich die Endstränge in dieser Hinsicht unrentabel, wenn überhaupt von einer Rentabilität gesprochen werden kann. Hingegen werden bei grösseren Rohrkalibern von zum Beispiel $100 \mathrm{~cm}$ und darüber die Transportkosten pro Sekundenliter Abwasser angenähert konstant, so dass sich bei grösseren Profilen als $\varnothing \mathrm{roO} \mathrm{cm}$ wirtschaftlich nicht mehr viel herausholen lässt.

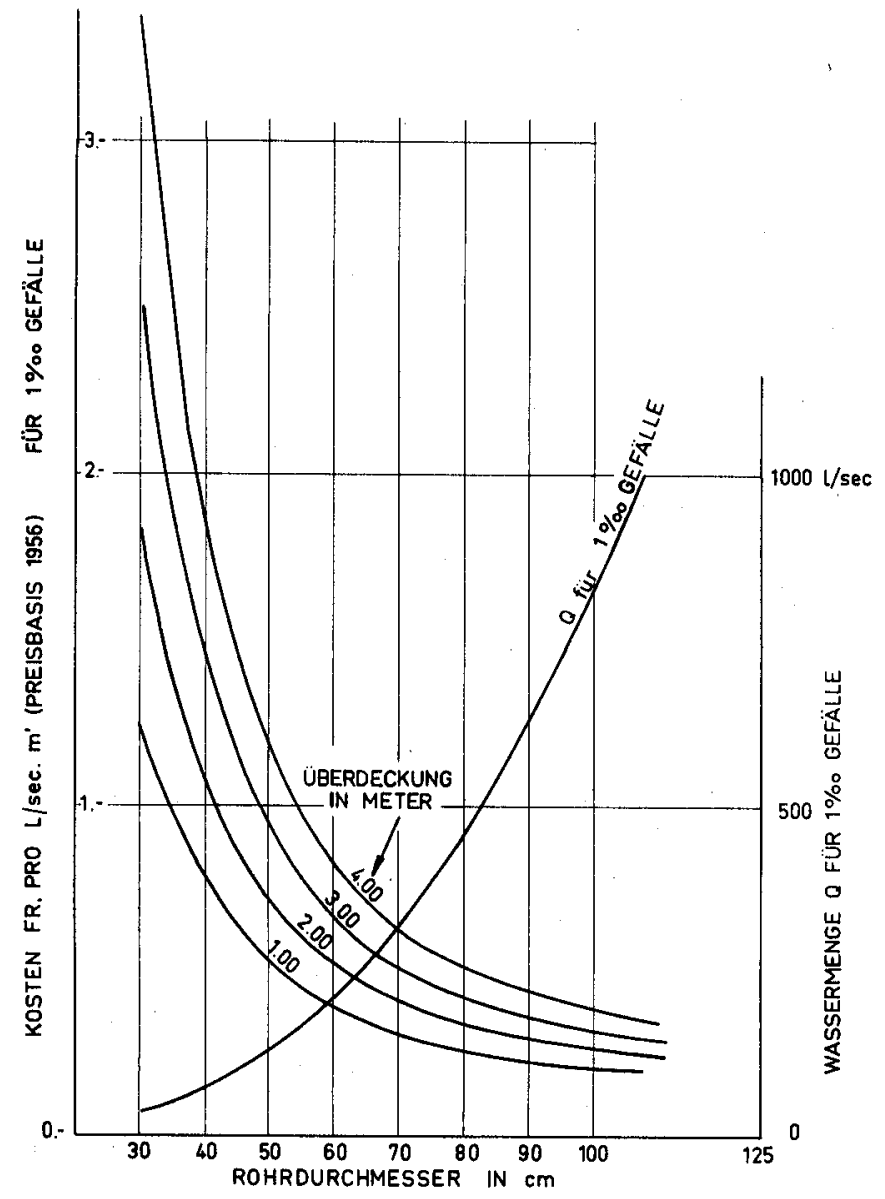

Abbildung 16

Kosten der Kanalisationen zur Ableitung von 1 1/s Abwasser bei einem Gefälle von $1 \%$ für verschiedene Rohrdurchmesser und bei verschiedenen Kanalïberdeckungen. 


\section{WIRKUNG DER REgENAUSLÄSSE AUF DIE VORFLUTER}

Die Berechnung, Dimensionierung und konstruktive Ausbildung der verschiedenen Regenauslässe ist ein äusserst umfangreiches Gebiet. Ich beschränke mich deshalb auf ein Teilgebiet, das meines Wissens noch wenig behandelt wurde, nämlich die Wirkung der Regenauslässe auf die Vorfluter. Anhand von zwei Diagrammen sei dargelegt, welche Belastung ein Vorfluter bei verschiedenen Mischungs- oder Verdünnungsverhältnissen erfährt in bezug auf die gesamte Verschmutzung, die bei direkter Einleitung des gesamten Abwassers in den Vorfluter am Orte des Regenauslasses entstehen würde. Zudem soll die Verbesserung der Belastungsverhältnisse errechnet werden, die bei zusätzlicher Erstellung von Regenwasserklärbecken bei den Regenauslässen erzielt werden können. Diese Belastungsverhältnisse werden sowohl über den biochemischen Sauerstoffbedarf als auch über den Gehalt an absetzbaren Stoffen abgewickelt.

Die Berechnungen fussen auf folgenden Grundlagen:

I. Das Einzugsgebiet des Regenwassers weise eine mittlere Besiedlungsdichte von I5O E/ha, einen Scheitelabflussbeiwert von 0,35 und einen Gesamtabflussbeiwert von 0,50 auf.

2. Der mittlere Abwasseranfall betrage 3501 pro Einwohner und Tag $(0,004 \mathrm{l} / \mathrm{s} \mathrm{E})$.

3. Der Trockenwetteranfall entspreche einem Abwasseranfall von 450 1/ET auf I4 Stunden bzw. von 550 1/ET auf I6 Stunden verteilt (0,009 1/s E).

4. Der Anteil und die Verteilung des Regenwasseranfalles entsprechen der für Zürich ermittelten Dauerkurve der Regenintensitäten [I 5 ].

5. Die Abwasserkonzentration sei bei Trocken- und Regenwetter konstant.

Die letztere Bedingung bedarf der Erläuterung.

Berechnungen mit sogenannten Verdünnungen unter der Annahme eines biochemischen Sauerstoff bedarfs des Regenwassers von o $\mathrm{mg} / \mathrm{l}$ wurden in der Literatur [ $\mathrm{I} 6]$ veröffentlicht. Solche Berechnungen ergeben jedoch bezüglich der Vorfluterbelastung ein wesentlich günstigeres Bild, als wenn gleiche Konzentration bei Regen- und bei Trockenwetter angenommen wird. Auf welche Grundlage stützt sich jedoch unseren Annahme? Über die Verunreinigung eines Vorfluters durch Regenmasser allein hat WILKINSON [I7] interessante Ergebnisse veröffentlicht. Ein im Trennsystem entwässertes Gebiet von 247,5 ha und 12500 Einwohnern wurde während eines Jahres im Hinblick auf die Regenwasserabflüsse und deren Zusammensetzung untersucht. Die Ergebnisse dieser Erhebungen lassen sich etwa wie folgt zusammenfassen. 
Die Laufzeit des Regenwassers bis zur Probenahmestelle betrug 19,2 Minuten. Die Konzentration des Regenwassers nach 20-30 Minuten Regendauer nahm in der Regel rasch ab. Der $\mathrm{BSB}_{5}$ betrug etwa $8 \mathrm{mg} / 1+3 \mathrm{mg} / 1$ für je 24 Stunden Trockenzeit zwischen zwei Regenfällen, im Maximum Ioo mg/l. An absetzbaren Stoffen betrug der Höchstwert $2045 \mathrm{mg} / \mathrm{l}$, der Mittelwert $358 \mathrm{mg} / \mathrm{l}$, mit einem Anteil an organischen Stoffen von $27 \%$.

Wenn schon das Regenwasser nicht als rein angesprochen werden kann, so ist dies bei der Entwässerung im Mischsystem für das Mischwasser

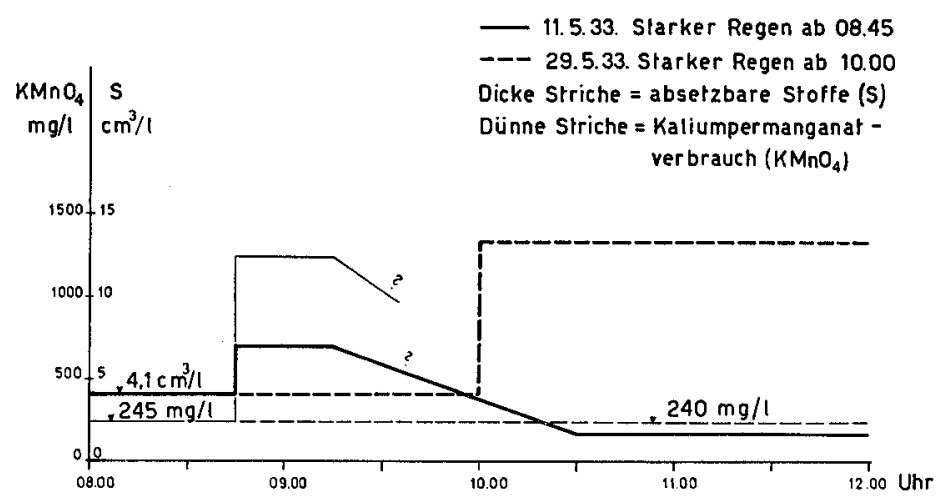

Hauplregenauslass bei Kläranlage Werdhölzli,Zürich (nach Brentano)

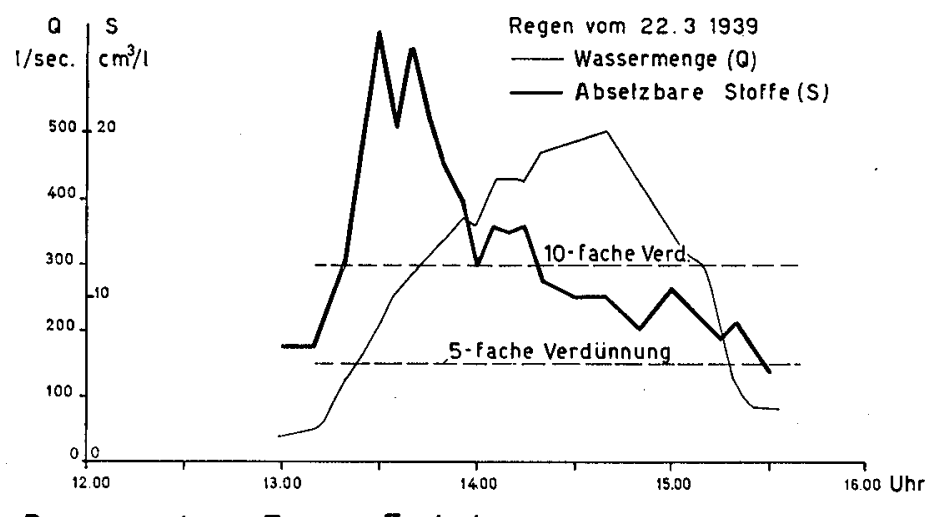

Regenauslass Essen-Frohnhausen (nach Müller-Neuhaus)

Abbildung 17

Abwasserkonzentration bei Regenwasserentlastungen. 
noch weniger der Fall. Namentlich in Flachgebieten und bei grösseren Siedlungsgebieten mit langen Laufzeiten wird die Beobachtung gemacht, dass die Abwasserkonzentration bei Regenwetter sogar ein Mehrfaches des Trockenwetteranfalles betragen kann. Dies wird durch Erhebungen bestätigt, die durch BrEnTANo [I8] und MüLLER-NeuHAus [I9] ausgeführt worden sind. In Abbildung 17 sind diese Erhebungen zusammengestellt.

Da bei längerer Regendauer die Kanäle offenbar reingespült sind und somit die Abflusskonzentration wieder zurückgeht, dürfte es nicht notwendig sein, im Mittel mit höheren Konzentrationen zu rechnen als der Trockenwetterkonzentration entspricht. Umgekehrt wird für das Entlastungswasser bei Städten auch nicht mit wesentlich geringeren Konzentrationen gerechnet werden dürfen. IMHOFF [Io] gibt an, dass durch den Einbau von Regenwasserklärbecken der Schlammanfall um rund $20 \%$ vergrössert werde. Da der Anfall von Regenwasser grössenordnungsmässig etwa $20 \%$ des Trockenwetteranfalls entspricht, so ergibt sich also auch nach der Feststellung von IMHOFF, dass die Konzentration an Schmutzstoffen im Mischwasser bei Regenfällen ungefähr von gleicher Grössenordnung sein dürfte wie beim Trockenwetter.

Unter den oben erwähnten Annahmen beträgt der gesamte Schmutzstoffanfall pro Hektare und Jahr, gemessen am biocbemischen Sauerstoffbedarf $\left(\mathrm{BSB}_{5}\right), 5250 \mathrm{~kg}$ bei den gewählten Berechnungsgrundlagen. Davon gelangen theoretisch zur Entlastung:

\begin{tabular}{|c|c|c|c|}
\hline $\begin{array}{c}\text { Entlastungs- } \\
\text { verhältnis }\end{array}$ & $\begin{array}{c}\mathrm{BSB}_{5} \\
\mathbf{k g}\end{array}$ & & $\begin{array}{c}\text { in \% des } \\
\text { Gesamtanfalls }\end{array}$ \\
\hline $1+0$ & 640 & & oder \\
$1+1$ & 323 & & 12,2 \\
$1+2$ & 206 & & 3,2 \\
$1+3$ & 151 & & 2,9 \\
$1+4$ & 120 & & 2,3 \\
$1+5$ & 103 & & 2,0 \\
$1+10$ & 65 & & $\mathbf{1 , 2}$ \\
\hline
\end{tabular}

Wird ein Entlastungsverhältnis von $\mathrm{I}+2$ (I Teil Trockenwetter- +2 Teile Regenwasseranfall) zugebilligt, zugleich jedoch die spätere Nachschaltung eines Regenwasserklärbeckens von Io Minuten Absetzzeit bei einer kritischen Regenintensität von $151 /$ sha verlangt, so kann der Vorfluter damit um $86 \mathrm{~kg} \mathrm{BSB}_{5} /$ ha Einzugsgebiet jährlich entlastet werden. Die Wirkung des Regenwasserklärbeckens bei einem Inhalt von $9 \mathrm{~m}^{3}$ pro 


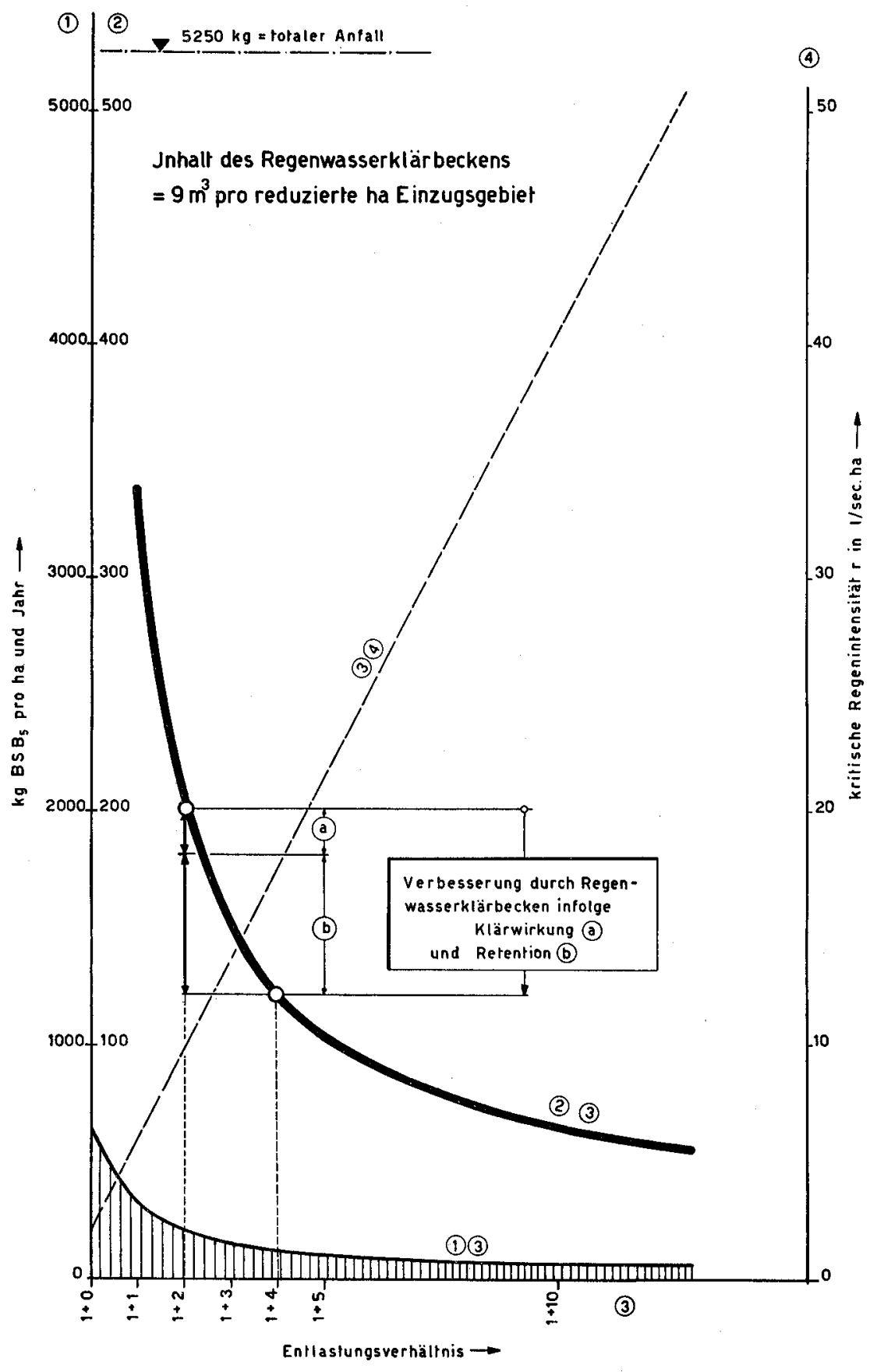

Abbildung 18

Vorfluterbelastung bezüglich $\mathrm{BSB}_{5}$ bei verschiedenen Entlastungsverhältnissen. Vergleich einer Entlastung 1+2 mit Regenwasserklärbecken mit einer Entlastung ohne Regenwasserklärbecken. 


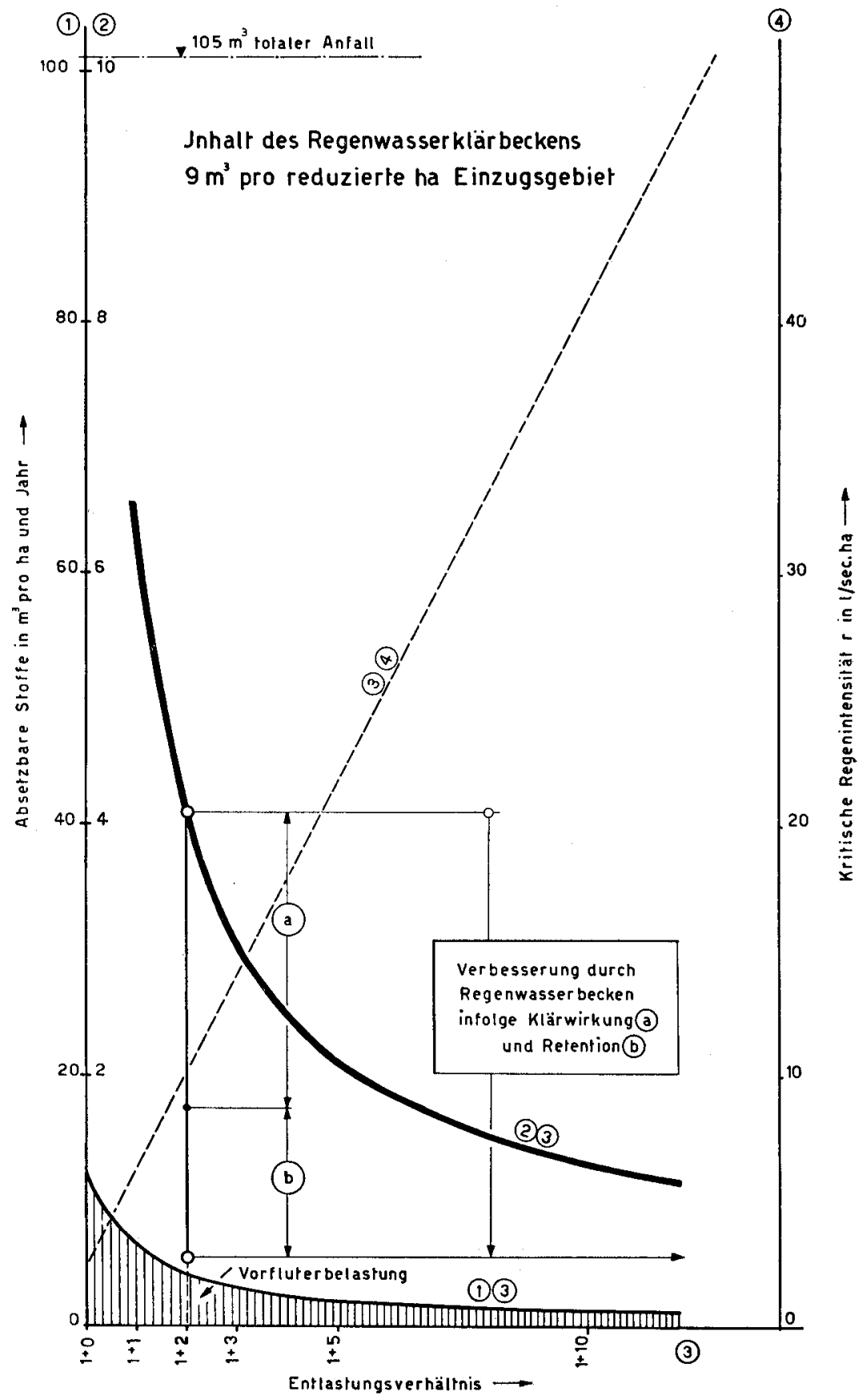

Abbildung 19

Vorfluterbelastung bezüglich absetzbarer Stoffe bei verschiedenen Entlastungsverhältnissen. Vergleich einer Entlastung 1+2 mit Regenwasserklärbecken mit einer Entlastung ohne Regenwasserklärbecken. 
reduzierte Hektare Einzugsgebiet entspricht somit theoretisch, gemessen am biochemischen Sauerstoff bedarf, einer Entlastung bei I +4 . Dabei erfolgt die Verbesserung zu 30\% durch die Klärwirkung des Beckens und zu 70\% infolge dessen Rückhaltevermögens (Abb. I 8). Daraus ergibt sich die grosse Überlegenheit der Regenwasserklärbecken gegenüber anderen Massnahmen (wie Rechen oder Sieben), sofern der Beurteilung der $\mathrm{BSB}_{5}$ zugrunde gelegt wird.

Wird als Maßstab unserer Betrachtungen nicht der biochemische Sauerstoff bedarf, sondern der Gehalt an absetzbaren Stoffen gewählt, so beträgt der Anfall an absetzbaren Stoffen pro Hektare und Jahr für das gewählte Beispiel $\operatorname{Io} \mathrm{m}^{3}$. Davon gelangen zur Entlastung, das heisst in den Vorfluter:

\begin{tabular}{|c|r|}
\hline $\begin{array}{c}\text { Entlastungs- } \\
\text { verhältnis }\end{array}$ & $\mathrm{m}^{3}$ \\
\hline $1+0$ & 12,8 \\
$1+1$ & 6,5 \\
$1+2$ & 4,1 \\
$1+3$ & 3,0 \\
$1+4$ & 2,4 \\
$1+5$ & 2,1 \\
$1+10$ & 1,3 \\
\hline
\end{tabular}

Wird wieder ein Entlastungsverhältnis von $\mathrm{I}+2$ bei Nachschaltung eines Regenwasserklärbeckens in der nämlichen Grössenordnung zugelassen, so würde dies etwa einer Vergrösserung des Entlastungsverhältnisses von $I+2$ auf über $I+20$ entsprechen, da die Menge der zurückgehaltenen Stoffe im Absetzbecken $3,6 \mathrm{~m}^{3} / \mathrm{ha} / \mathrm{Jahr}$ betragen würde (Abb. 19). Von dieser Menge entfallen 66\% auf die Klärwirkung und rund $33 \%$ auf das Rückhaltevermögen oder die Retention des Klärbeckens.

Vorstehende Überlegungen zeigen aurch, dass es̀ wenig Sinn hat, namentlich bei Seen und Stauanlagen, möglichst hohe Entlastungsverhältnisse zur Verminderung der Schlammablagerungen im Stausee vorzuschreiben. In dieser Hinsicht ist die Nachschaltung von Regenwasserklärbecken wirksamer. 


\section{Welcher Anteil an Regenwasser soll biologisch behandelt werden?}

\section{A. Problemstellung}

In der Abwassertechnik ist es gebräuchlich, neben dem Trockenwetteranfall zusätzlich das 2- bis 4 fache an Regenwasser in den mechanischen Teil einer Kläranlage zu übernehmen und mitzuklären $(I+2$ bis $I+4)$, sofern das Entwässerungsnetz nach dem Mischsystem angeordnet wird. Zwischen dem Absetzbecken und dem biologischen Teil der Reinigungsanlage wird gewöhnlich eine Regenentlastung angeordnet, so dass der biologische Teil nur eine Beschickung in der Grössenordnung des Trockenwetteranfalles erhält $(\mathrm{I}+\mathrm{O})$. Je nach der Definition des Trockenwetteranfalles, der in einzelnen Ländern verschieden, meist jedoch nur unklar definiert ist und dessen genaue Festlegung im Hinblick auf die eigentliche Bedeutung dieses Begriffes als dringlich erscheint, kann es vorkommen, dass bei einer Beschickung der biologischen Anlage mit dem Trockenwetteranfall allein zu gewissen Tagesstunden an Höchstverbrauchstagen Abwasserspitzen ohne biologische Reinigung, nur mechanisch geklärt, in den Vorfluter gelangen.

Dieser unerwünschten Erscheinung zu steuern, wählt man in der Schweiz trotz vorsichtigster Wahl des Begriffs «Trockenwetteranfall» als Belastung der biologischen Anlage häufig den $\mathrm{r}, 5$ fachen Trockenwetteranfall $(\mathrm{I}+0,5)$, wobei speziell für Kläranlagen an Seen die Tendenz besteht, den doppelten Trockenwetteranfall biologisch zu behandeln. In ge-
wissen Kantonen werden seitens massgebender Fachleute Vorstösse unternommen, bei Regenwetter noch grössere Regenwasseranteile mitzubehandeln, so dass auch der biologische Teil der Anlage eine Belastung von I +2 und mehr erhalten würde.

\section{B. KONSEQUENZEN AUS DIESER FORDERUNG}

Das Durchschleusen höherer Regenwasseranteile durch den biologischen Teil einer Reinigungsanlage kann, sofern nur die Verbindungskanäle der Becken bei Belebtschlammanlagen und die Abwasserrückgabe in den Vorfluter entsprechend der grösseren sekundlichen Belastung bemessen werden, mit relativ geringfügigen zusätzlichen finanziellen Mitteln erfolgen. Bei Tropfkörperanlagen kommt jedoch bereits eine Vergrösserung des Abwasserpumpwerkes samt Armaturen und Druckleitungen in Frage. 
Die grösste Sorge bereitet jedoch das Nachklärbecken. Je nach der Grösse der Sicherheitsmarge gegen Überlastungen, mit der das Nachklärbecken bemessen ist, kann ein mehr oder weniger grosser Regenwasseranteil mitgeklärt werden, ohne dass Kurzschlusserscheinungen mit mangelhafter Klärwirkung oder ein Auftreiben des sogenannten schwebenden Filters in den Nachklärbecken zustande kommt. Normalerweise wird aber eine solche Überlastung höchstens bis zu einer Belastung von $\mathrm{I}+0,5$, extrem I + I, noch zulässig sein. Werden grössere Regenwassermengen mitbehandelt, so sind unweigerlich eine Vergrösserung des Nachklärbeckens und damit erhebliche Mehrkosten in Kauf zu nehmen. Logischerweise sollten diese Mehrkosten einen entsprechend besseren Schutz des Vorfluters gewährleisten.

\section{WAS KANN DURCH BIOLOGISCHE REINIGUNG EINES ERHÖHTEN REGENWASSERANTEILS IM HINBLICK AUF DEN VORFLUTER ERREICHT WERDEN?}

Zur Beantwortung dieser Frage wurden an einem Beispiel die Vorfluterbelastungen, gemessen am biochemischen Sauerstoffbedarf, durchgerechnet, wie sie bei verschieden grossen Anteilen biologisch behandelten Regenwassers zu erwarten sind. Die Berechnungen fussen wieder auf der Annahme von Wohnzonen im Einzugsgebiet, wie sie in der Stadt Zürich für deren Aussengebiete vorwiegend erstellt werden: mit $1_{50} \mathrm{E} / \mathrm{ha}$, einem Scheitelabflussbeiwert von 0,35 und einem Gesamtabflussbeiwert von 0,50 . Zudem sind wieder die im Kapitel VII dargelegten Annahmen dem Beispiel zugrunde gelegt.

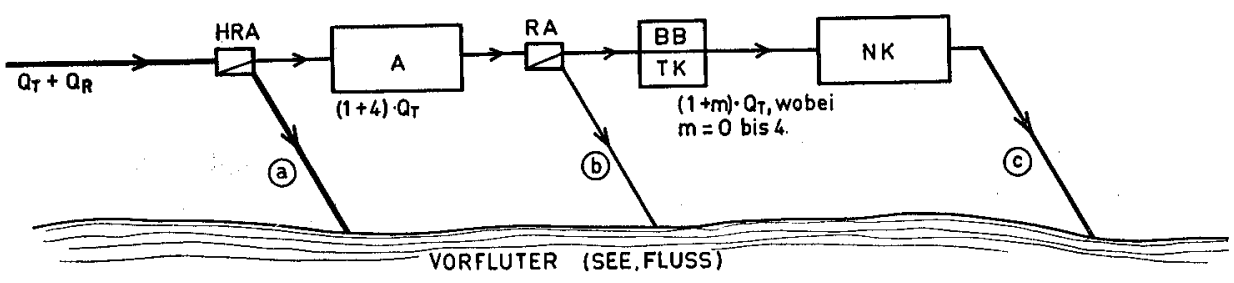

Abbildung 20

Schemaskizze einer Abwasserreinigungsanlage mit Angabe der Vorfluterbelastung bei verschiedenen Regenwasseranteilen $(1+m)$, die biologisch mitbehandelt werden sollen. 


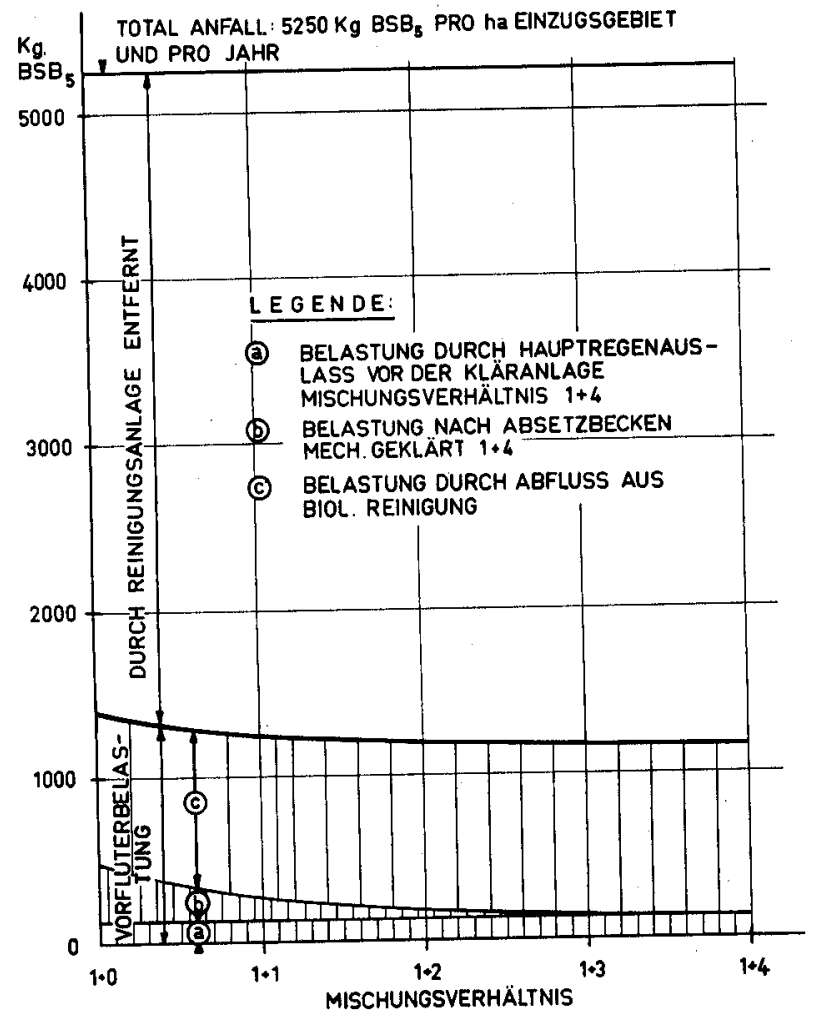

Abbildung 21

Vorfluterbelastung bei biologischer Teilreinigung $(\eta=70 \%)$ bei verschiedenen Regenwasseranteilen $(1+m)$, die biologisch mitbehandelt werden sollen.

Nach Abbildung 20 erfolgen die Vorfluterbelastungen aus dem Kläranlagegebiet an drei Stellen:

a) vom Hauptregenauslass vor der Kläranlage;

b) vom Auslass zwischen Absetzbecken und der biologischen Reinigung;

c) durch den Abfluss aus der biologischen Reinigung.

Die Grösse der Belastung des Vorfluters aus diesen drei Stellen wurde rechnerisch in Kilogramm BSB $_{5}$ pro Hektare Einzugsgebiet und pro Jahr für die Mischungsverhältnisse $\mathrm{I}+\mathrm{O}$ bis $\mathrm{I}+4$ ermittelt, die biologisch $z u$ behandeln sind, unter der Annahme, dass durch das Absetzbecken in allen Fällen $\mathrm{x}+4$ Trockenwetteranteile durchgeleitet werden. Zudem wurden die Berechnungen für Teilreinigung und Vollreinigung (Reinigungseffekt 


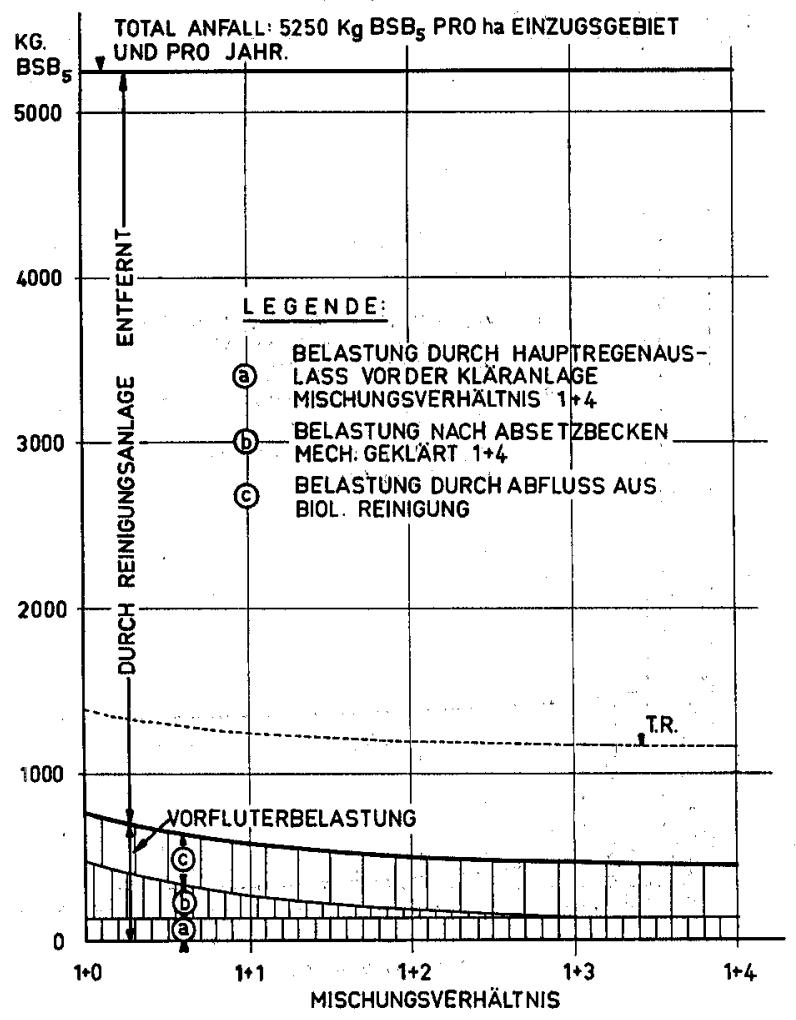

Abbildung 22

Vorfluterbelastung bei biologischer Vollreinigung $(\eta=90 \%)$ bei verschiedenen Regenwasseranteilen $(1+m)$, die biologisch mitbehandelt werden sollen. Die gestrichelte Linie T. R. entspricht der Vorfluterbelastung bei biologischer Teilreinigung.

gegenüber dem mechanisch geklärten $A$ bwasser $=70 \%$ bzw. 90\%) durchgeführt. Die Ergebnisse dieser Ermittlungen sind graphisch in den Abbildungen $2 \mathrm{I}$ und 22 dargestellt.

Aus diesen Abbildungen ist zu entnehmen, dass mit der Durchschleusung höherer Regenwasseranteile, als etwa der Mischung I + I entspricht - immer gemessen am $\mathrm{BSB}_{5}$-, namentlich bei Teilreinigung praktisch für den Vorfluter beinahe nichts erreicht wird. Diese Schlussfolgerung wird noch verschärft durch den Umstand, dass vorliegende Berechnungen unter der Annahme konstanter Klär- und Reinigungseffekte im Absetzbecken, biologischen Teil und im Nachklärbecken durchgeführt wurden, unabhängig von der Grösse der Abwassermenge. Da jedoch diese Effekte mit 
den höheren Mischungszahlen tatsächlich schlechter werden, so ist sogar eine Mehrbelastung des Vorfluters bei biologischer Mitbehandlung grösserer Regenwasseranteile nicht ausgeschlossen. Da dies unter allen Umständen vermieden werden soll, bleibt nichts anderes übrig, als namentlich den Inhalt des Nachklärbeckens wesentlich zu vergrössern, ohne damit jedoch eine in wirtschaftlicher Hinsicht befriedigende Entlastung des Vorfluters zu erzielen. Sollten in bestimmten Gebieten die Verhältnisse dafür sprechen, dass die Verdünnungstheorie bei Regenwetter mit grosser Wahrscheinlichkeit angenähert zutrifft (relativ kleine Einzugsgebiete in hügeligem Gelände), dann würden obige Schlussfolgerungen in noch vermehrtem Masse gelten.

Die durchgeführten Studien und vorstehende Überlegungen zeigen, dass es - $\mathrm{am} \mathrm{BSB}_{5}$ beurteilt - keinen Sinn hat, grössere Regenwasseranteile als $I+0,5$, aller extremsten Falles $I+I$, biologisch zu behandeln. Sofern ein Vorfluter vermehrten Schutz erfordert, so ist die Lösung nicht in einer biologischen Behandlung grösserer Regenwasseranteile zu suchen, sondern in einer Vergrösserung des Reinigungseffektes der biologischen Anlage. Überschlägige Berechnungen zeigen, dass durch eine geringfügige Verbesserung des Reinigungseffektes für den Vorfluter, namentlich bei einem See, mehr erreicht wird als mit einer wesentlichen Vergrösserung der Nachklärbecken bei Mitbehandlung erheblicher Regenwassermengen. Wesentlich ist, dass sich eine Verbesserung des Reinigungseffektes während des ganzen Jahres auswirkt, während erhebliche Regenwasseranteile nur stundenweise, höchstens tageweise, auftreten und sich somit deren Reinigung nur über eine relativ kleine Zeitspanne auswirken kann. Statt somit das Nachklärbecken zum Beispiel zu verdoppeln, also um $100 \% \mathrm{zu}$ vergrössern, erweitert man in der Regel zweckmässiger den eigentlichen biologischen Teil, das heisst die Belüftungsbecken oder Tropfkörper um etwa $5-10 \%$ ihrer Volumen und erzielt damit bedeutend wirtschaftlicher im Vorfluter die gleichen Effekte.

Ich bin mir vollständig bewusst, dass vorstehende Berechnungen nicht ohne weiteres verallgemeinert werden dürfen, dass diese ebenfalls über den Stickstoff und den Phosphor abgewickelt werden müssen und dass eigentlich jeder vorliegende Fall für sich durchgerechnet werden sollte, wobei auch dann noch Fehlerquellen auftreten können, die in der Unvollkommenheit der vorhandenen Grundlagen liegen, Dies sollte jedoch weder bei diesen Fragen noch in der gesamten Abwassertechnik der Anlass dazu sein, von jeglichen Berechnungen abzusehen. 


\section{LITERA'TURVERZEICHNIS}

[I] D. KEHR, Standortfragen von Kläranlagen, erschienen als Abschnitt CCV der Stadtreinigung (Verlag. Technik und Kultur, Berlin 1955).

[2] E. BAROCKA, Wirtschaftliche, organisatorische und finanzielle Probleme des Wasserbaus und des Meliorationswesens (Verlag Oldenbourg, München 1953), S. 693.

[3] P. WILDI, Die Notwendigkeit genereller Kanalisationsprojekte und ibre Grundlage, in: Gewässerschutz und Abwasserreinigungsanlagen (Verlag Schweiz. Baublatt AG., Rüschlikon-Zürich I952), S. 78.

[4] H. AdDisen, A Treatise on Applied Hydraulics (London I954), S. 65.

[s] H. E. BabBitT, Seperage and Semage Treatment, 7. Aufl. (New York I953), S. 386.

[6] J. H. Ludwig und R. G. Ludwig, Design of Palmer-Bowlus Flumes, SIW 23, No. 9 (Sept.), 1096 (195I).

[7] A. KROPF, Beitrag zum Sandfangproblem (Zürich I946).

[8] A. Kafaghi, Der Venturikanal (Theorie und Anwendung), Mitteilungen der Versuchsanstalt für Wasserbau (Zürich I942).

[9] v. D. EMDE, Messtecbnik im Abrassermesen kleinerer und mittlerer Städte, ATV-Bericht, Heft 6 (München I955), S. 280.

[1о] K. IMHOFF, Tascbenbuch der Stadtentwässerung, I6. Aufl. (München).

[ir] L. G. Demidow und G. G. Schigorin, Kanalisation, Zweiter Teil: Abwasserreinigung (Leipzig 1955), S. 229.

[12] W. GEISSLER, Kanalisation und Abmasserreinigung (Berlin 1933).

[r3] L. G. Demidow und G. G. Schigorin, Kanalisation, Erster Teil: Das Kanalisationsnetz (Leipzig I953), S. SI.

[14] TH. HEYD, Die Wirtscbaftlicbkeit bei den Stadtentmässerungsverfabren (Mannheim I908), S. 78.

[Is] A. Hörler, Die Wirkung der Regenauslässe, S. B. Z. 118, Nr. 20 (I5. Nov. I94I).

[I6] B. BAYERLE, Die Verschmutzung der Wasserläufe durch die Regenauslässe der Ent wässerungsnetze, Gesundheitsing. 46, H. 7 und 8,73 und 85 (1923).

[I7] R. WILKINSON, The Quality of Rainfall Run-OffW ater from a Housing Estate, J. Inst. publ. Hlth. Eng., London 1956 (April), 70.

[18] H. BRENTANo, Untersucbungen über die Abwasserperbältnisse von Züricb (Solothurn I934).

[I9] G. MüLler-Neuhaus, Zur Frage der Bemessung pon Regenüberfällen bei Entwässerungsnetzen und Kläranlagen, Gesundheitsing. 71, H. 9/10, 149 (1950). 